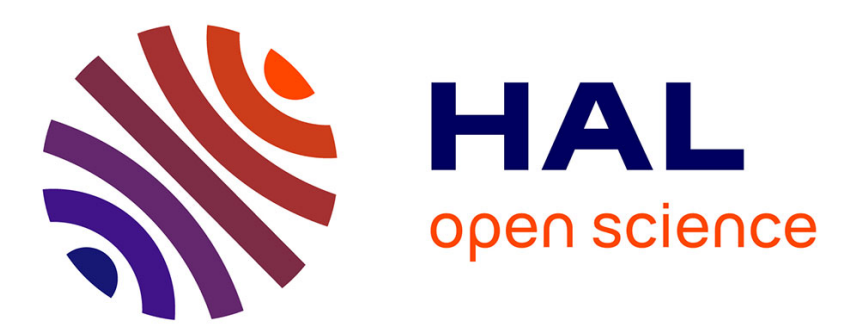

\title{
Variational analysis for options with stochastic volatility and multiple factors
}

Joseph Frédéric Bonnans, Axel Kröner

\section{To cite this version:}

Joseph Frédéric Bonnans, Axel Kröner. Variational analysis for options with stochastic volatility and multiple factors. SIAM Journal on Financial Mathematics, 2018, 9 (2), pp.465-492. hal-01516011v3

\section{HAL Id: hal-01516011 \\ https://hal.inria.fr/hal-01516011v3}

Submitted on 9 Apr 2018

HAL is a multi-disciplinary open access archive for the deposit and dissemination of scientific research documents, whether they are published or not. The documents may come from teaching and research institutions in France or abroad, or from public or private research centers.
L'archive ouverte pluridisciplinaire HAL, est destinée au dépôt et à la diffusion de documents scientifiques de niveau recherche, publiés ou non, émanant des établissements d'enseignement et de recherche français ou étrangers, des laboratoires publics ou privés. 


\title{
VARIATIONAL ANALYSIS FOR OPTIONS WITH STOCHASTIC VOLATILITY AND MULTIPLE FACTORS*
}

\author{
J. FRÉDÉRIC BONNANS ${ }^{\dagger}$ AND AXEL KRÖNER ${ }^{\ddagger}$
}

\begin{abstract}
This paper performs a variational analysis for a class of European or American options with stochastic volatility models, including those of Heston and Achdou-Tchou. Taking into account partial correlations and the presence of multiple factors, we obtain the well-posedness of the related partial differential equations, in some weighted Sobolev spaces. This involves a generalization of the commutator analysis introduced by Achdou and Tchou in [3].
\end{abstract}

Key words. finance, options, partial differential equations, variational formulation, parabolic variational inequalities

AMS subject classifications. 35K20, 35K $85,91 \mathrm{G} 80$

1. Introduction. In this paper we consider variational analysis for the partial differential equations associated with the pricing of European or American options. For an introduction to these models, see Fouque et al., [11]. We will set up a general framework of variable volatility models, which is in particular applicable on the following standard models which are well established in mathematical finance. The well-posedness of PDE formulations of variable volatility poblems was studied in $[2,3,1,18]$, and in the recent work $[9,10]$.

Let the $W_{i}(t)$ be Brownian motions on a filtered probability space. The variable $s$ denotes a financial asset, and the components of $y$ are factors that influence the volatility:

(i) The Achdou-Tchou model [3], see also Achdou, Franchi, and Tchou [1]:

$$
\left\{\begin{array}{l}
\mathrm{d} s(t)=r s(t) \mathrm{d} t+\sigma(y(t)) s(t) \mathrm{d} W_{1}(t), \\
\mathrm{d} y(t)=\theta(\mu-y(t)) \mathrm{d} t+\nu \mathrm{d} W_{2}(t)
\end{array}\right.
$$

with the interest rate $r$, the volatility coefficient $\sigma$ function of the factor $y$ whose dynamics involves a parameter $\nu>0$, and positive constants $\theta$ and $\mu$.

(ii) The Heston model [14]

$$
\left\{\begin{array}{l}
\mathrm{d} s(t)=s(t)\left(r \mathrm{~d} t+\sqrt{y(t)} \mathrm{d} W_{1}(t)\right) \\
\mathrm{d} y(t)=\theta(\mu-y(t)) \mathrm{d} t+\nu \sqrt{y(t)} \mathrm{d} W_{2}(t) .
\end{array}\right.
$$

(iii) The Double Heston model, see Christoffersen, Heston and Jacobs [17], and also Gauthier and Possamaï [12]:

$$
\left\{\begin{array}{l}
\mathrm{d} s(t)=s(t)\left(r \mathrm{~d} t+\sqrt{y_{1}(t)} \mathrm{d} W_{1}(t)+\sqrt{y_{2}(t)} \mathrm{d} W_{2}(t)\right), \\
\mathrm{d} y_{1}(t)=\theta_{1}\left(\mu_{1}-y_{1}(t)\right) \mathrm{d} t+\nu_{1} \sqrt{y_{1}(t)} \mathrm{d} W_{3}(t), \\
\mathrm{d} y_{2}(t)=\theta_{2}\left(\mu_{2}-y_{2}(t)\right) \mathrm{d} t+\nu_{2} \sqrt{y_{2}(t)} \mathrm{d} W_{4}(t) .
\end{array}\right.
$$

\footnotetext{
*Submitted to the editors DATE.
}

Funding: The first author was suported by the Laboratoire de Finance des Marchés de l'Energie, Paris, France. Both authors were supported by a public grant as part of the Investissement d'avenir project, reference ANR-11-LABX-0056-LMH, LabEx LMH, in a joint call with Gaspard Monge Program for optimization, operations research and their interactions with data sciences.

${ }^{\dagger}$ CMAP, Ecole Polytechnique, CNRS, Université Paris Saclay, and Inria, France (Frederic.Bonnans@inria.fr).

${ }^{\ddagger}$ Department of Mathematics, Humboldt University of Berlin, Berlin, Germany; CMAP, Ecole Polytechnique, CNRS, Université Paris Saclay, and Inria, France (axel.kroener@math.hu-berlin.de) 
In the last two models we have similar interpretations of the coefficients; in the double Heston model, denoting by $\langle\cdot, \cdot\rangle$ the correlation coefficients, we assume that there are correlations only between $W_{1}$ and $W_{3}$, and $W_{2}$ and $W_{4}$. Consider now the general multiple factor model

$$
\begin{aligned}
\mathrm{d} s & =r s(t) \mathrm{d} t+\sum_{k=1}^{N} f_{k}\left(y_{k}(t)\right) s^{\beta_{k}}(t) \mathrm{d} W_{k}(t), \\
\mathrm{d} y_{k} & =\theta_{k}\left(\mu_{k}-y_{k}(t)\right) \mathrm{d} t+g_{k}\left(y_{k}(t)\right) \mathrm{d} W_{N+k}(t), \quad k=1, \ldots, N .
\end{aligned}
$$

Here the $y_{k}$ are volatility factors, $f_{k}\left(y_{k}\right)$ represents the volatility coefficient due to $y_{k}, g_{k}\left(y_{k}\right)$ is a volatility coefficient in the dynamics of the $k$ th factor with positive constants $\theta_{k}$ and $\mu_{k}$. Let us denote the correlation between the $i$ th and $j$ th Brownian motions by $\kappa_{i j}$ : this is a measurable function of $(s, y, t)$ with value in $[0,1]$ (here $s \in(0, \infty)$ and $y_{k}$ belongs to either $(0, \infty)$ or $\left.\mathbb{R}\right)$, see below. We asssume that we have nonzero correlations only between the Brownian motions $W_{k}$ and $W_{N+k}$, for $k=1$ to $N$, i.e.

$$
\kappa_{i j}=0 \quad \text { if } i \neq j \text { and }|j-i| \neq N .
$$

Note that, in some of the main results, we will assume for the sake of simplicity that the correlations are constant.

We apply the developed analysis to a subclass of stochastic volatility models, obtained by assuming that $\kappa$ is constant and

$$
\left|f_{k}\left(y_{k}\right)\right|=\left|y_{k}\right|^{\gamma_{k}} ;\left|g_{k}\left(y_{k}\right)\right|=\nu_{k}\left|y_{k}\right|^{1-\gamma_{k}} ; \quad \beta_{k} \in(0,1] ; \quad \nu_{k}>0 ; \quad \gamma_{k} \in(0, \infty) .
$$

This covers in particular a variant of the Achdou and Tchou model with multiple factors (VAT), when $\gamma_{k}=1$, as well as a generalized multiple factor Heston model (GMH), when $\gamma_{k}=1 / 2$, i.e., for $k=1$ to $N$ :

$$
\begin{array}{lll}
\text { VAT: } & f_{k}\left(y_{k}\right)=y_{k}, & g_{k}\left(y_{k}\right)=\nu_{k}, \\
\text { GMH: } & f_{k}\left(y_{k}\right)=\sqrt{y}_{k}, & g_{k}\left(y_{k}\right)=\nu_{k} \sqrt{y}_{k} .
\end{array}
$$

For a general class of stochastic volatility models with correlation we refer to Lions and Musiela [16].

The main contribution of this paper is variational analysis for the pricing equation corresponding to the above general class in the sense of the Feynman-Kac theory. This requires in particular to prove continuity and coercivity properties of the corresponding bilinear form in weighted Sobolev spaces $H$ and $V$, respectively, which have the Gelfand property and allow the application of the Lions and Magenes theory [15] recalled in Appendix A and the regularity theory for parabolic variational inequalities recalled in Appendix B. A special emphasis is given to the continuity analysis of the rate term in the pricing equation. Two approaches are presented, the standard one and an extension of the one based on the commutator of first-order differential operators as in Achdou and Tchou [3], extended to the Heston model setting by Achdou and Pironneau [18]. Our main result is that the commutator analysis gives stronger results for the subclass defined by (1.6), generalizing the particular cases of the VAT and GMH classes, see remarks 6.2 and 6.4. In particular we extend some of the results by [3].

This paper is organized as follows. In section 2 we give the expression of the bilinear form associated with the original PDE, and check the hypotheses of continuity and semi-coercivity of this bilinear form. In section 3 we show how to refine this analysis by taking into account the commutators of the first-order differential operators 
associated with the variational formulation. In section 4 we show how to compute the weighting function involved in the bilinear form. In section 5 we develop the results for a general class introduced in the next section. In section 6 we specialize the results to stochastic volatility models. The appendix recalls the main results of the variational theory for parabolic equations, with a discussion on the characterization of the $V$ functional spaces in the case of one dimensional problems.

Notation. We assume that the domain $\Omega$ of the PDEs to be considered in the sequel of this paper has the following structure. Let $(I, J)$ be a partition of $\{0, \ldots, N\}$, with $0 \in J$, and

$$
\Omega:=\prod_{k=0}^{N} \Omega_{k} ; \quad \text { with } \quad \Omega_{k}:= \begin{cases}\mathbb{R} & \text { when } k \in I, \\ (0, \infty) & \text { when } k \in J .\end{cases}
$$

Let $L^{0}(\Omega)$ denote the space of measurable functions over $\Omega$. For a given weighting function $\rho: \Omega \rightarrow \mathbb{R}$ of class $C^{1}$, with positive values, we define the weighted space

$$
L^{2, \rho}(\Omega):=\left\{v \in L^{0}(\Omega) ; \int_{\Omega} v(x)^{2} \rho(x) \mathrm{d} x<\infty\right\},
$$

which is a Hilbert space endowed with the norm

$$
\|v\|_{\rho}:=\left(\int_{\Omega} v(x)^{2} \rho(x) \mathrm{d} x\right)^{1 / 2} .
$$

By $\mathcal{D}(\Omega)$ we denote the space of $C^{\infty}$ functions with compact support in $\Omega$. By $H_{l o c}^{2}(\Omega)$ we denote the space of functions over $\Omega$ whose product with an element of $\mathcal{D}(\Omega)$ belongs to the Sobolev space $H^{2}(\Omega)$.

Besides, let $\Phi$ be a vector field over $\Omega$ (i.e., a mapping $\Omega \rightarrow \mathbb{R}^{n}$ ). The first-order differential operator associated with $\Phi$ is, for $u: \Omega \rightarrow \mathbb{R}$ the function over $\Omega$ defined by

$$
\Phi[u](x):=\sum_{i=0}^{n} \Phi_{i}(x) \frac{\partial u}{\partial x_{i}}(x), \quad \text { for all } x \in \Omega .
$$

2. General setting. Here we give compute the bilinear form associated with the original PDE, in the setting of the general multiple factor model (1.4). Then we will check the hypotheses of continuity and semi-coercivity of this bilinear form.

2.1. Variational formulation. We compute the bilinear form of the variational setting, taking into account a general weight function. We wil see how to choose the functional spaces for a given $\rho$, and then how to choose the weight itself.

2.1.1. The elliptic operator. In financial models the underlying is solution of stochastic differential equations of the form

$$
d X(t)=b(t, X(t)) \mathrm{d} t+\sum_{i=1}^{n_{\sigma}} \sigma_{i}(t, X(t)) d W_{i} .
$$

Here $X(t)$ takes values in $\Omega$, defined in (1.8). That is, $X_{1}$ corresponds to the $s$ variable, and $X_{k+1}$, for $k=1$ to $N$, corresponds to $y_{k}$. We have that $n_{\sigma}=2 N$.

So, $b$ and $\sigma_{i}$, for $i=1$ to $n_{\sigma}$, are mappings $(0, T) \times \Omega \rightarrow \mathbb{R}^{n}$, and the $W_{i}$, for $i=1$ to $n_{\sigma}$, are standard Brownian processes with correlation $\kappa_{i j}:(0, T) \times \Omega \rightarrow \mathbb{R}$ 
between $W_{i}$ and $W_{j}$ for $i, j \in\left\{1, \ldots, n_{\sigma}\right\}$. The $n_{\sigma} \times n_{\sigma}$ symmetric correlation matrix $\kappa(\cdot, \cdot)$ is nonnegative with unit diagonal:

$$
\kappa(t, x) \succeq 0 ; \quad \kappa_{i i}=1, i=1, \ldots, n_{\sigma}, \quad \text { for a.a. }(t, x) \in(0, T) \times \Omega .
$$

Here, for symmetric matrices $B$ and $C$ of same size, by " $C \succeq B$ " we mean that $C-B$ is positive semidefinite. The expression of the second order differential operator $A$ corresponding to the dynamics (2.1) is, skipping the time and space arguments, for $u:(0, T) \times \Omega \rightarrow \mathbb{R}:$

$$
A u:=r u-b \cdot \nabla u-\frac{1}{2} \sum_{i, j=1}^{n_{\sigma}} \kappa_{i j} \sigma_{j}^{\top} u_{x x} \sigma_{i},
$$

where

$$
\sigma_{j}^{\top} u_{x x} \sigma_{i}:=\sum_{k, \ell=1}^{n_{\sigma}} \sigma_{k j} \frac{\partial u^{2}}{\partial x_{k} \partial x_{\ell}} \sigma_{\ell i},
$$

$r(x, t)$ represents an interest rate, and $u_{x x}$ is the matrix of second derivatives in space of $u$. The associated backward PDE for a European option is of the form

$$
\left\{\begin{aligned}
-\dot{u}(t, x)+A(t, x) u(t, x) & =f(t, x), & & (t, x) \in(0, T) \times \Omega ; \\
u(x, T) & =u_{T}(x), & & x \in \Omega,
\end{aligned}\right.
$$

with $\dot{u}$ the notation for the time derivative of $u, u_{T}(x)$ payoff at final time (horizon) $T$ and the r.h.s. $f(t, x)$ represents dividends (often equal to zero).

In case of an American option we obtain a variational inequality; for details we refer to Appendix D.

2.1.2. The bilinear form. In the sequel we assume that

$$
b, \sigma, \kappa \text { are } C^{1} \text { mappings over }[0, T] \times \Omega \text {. }
$$

Multiplying (2.3) by the test function $v \in \mathcal{D}(\Omega)$ and the continuously differentiable weight function $\rho: \Omega \rightarrow \mathbb{R}$ and integrating over the domain we can integrate by parts; since $v \in \mathcal{D}(\Omega)$ there will be no contribution from the boundary. We obtain

$$
-\frac{1}{2} \int_{\Omega} \sigma_{j}^{\top} u_{x x} \sigma_{i} v \kappa_{i j} \rho=\sum_{p=0}^{3} a_{i j}^{p}(u, v),
$$

with

$$
a_{i j}^{0}(u, v):=\frac{1}{2} \int_{\Omega} \sum_{k, \ell=1}^{n} \sigma_{k j} \sigma_{\ell i} \frac{\partial u}{\partial x_{k}} \frac{\partial v}{\partial x_{\ell}} \kappa_{i j} \rho=\frac{1}{2} \int_{\Omega} \sigma_{j}[u] \sigma_{i}[v] \kappa_{i j} \rho,
$$

$$
a_{i j}^{1}(u, v):=\frac{1}{2} \int_{\Omega} \sum_{k, \ell=1}^{n} \sigma_{k j} \sigma_{\ell i} \frac{\partial u}{\partial x_{k}} \frac{\partial\left(\kappa_{i j} \rho\right)}{\partial x_{\ell}} v=\frac{1}{2} \int_{\Omega} \sigma_{j}[u] \sigma_{i}\left[\kappa_{i j} \rho\right] \frac{v}{\rho} \rho,
$$

$$
a_{i j}^{2}(u, v):=\frac{1}{2} \int_{\Omega} \sum_{k, \ell=1}^{n} \sigma_{k j} \frac{\partial\left(\sigma_{\ell i}\right)}{\partial x_{\ell}} \frac{\partial u}{\partial x_{k}} v \kappa_{i j} \rho=\frac{1}{2} \int_{\Omega} \sigma_{j}[u]\left(\operatorname{div} \sigma_{i}\right) v \kappa_{i j} \rho
$$




$$
a_{i j}^{3}(u, v):=\frac{1}{2} \int_{\Omega} \sum_{k, \ell=1}^{n} \frac{\partial\left(\sigma_{k j}\right)}{\partial x_{\ell}} \sigma_{\ell i} \frac{\partial u}{\partial x_{k}} v \kappa_{i j} \rho=\frac{1}{2} \int_{\Omega} \sum_{k=1}^{n} \sigma_{i}\left[\sigma_{k j}\right] \frac{\partial u}{\partial x_{k}} v \kappa_{i j} \rho .
$$

Also, for the contributions of the first and zero order terms resp. we get

$$
a^{4}(u, v):=-\int_{\Omega} b[u] v \rho ; \quad a^{5}(u, v):=\int_{\Omega} \operatorname{ruv} \rho .
$$

Set

$$
a^{p}:=\sum_{i, j=1}^{n_{\sigma}} a_{i j}^{p}, \quad p=0, \ldots, 3 .
$$

The bilinear form associated with the above PDE is

$$
a(u, v):=\sum_{p=0}^{5} a^{p}(u, v) .
$$

From the previous discussion we deduce that

Lemma 2.1. Let $u \in H_{\ell o c}^{2}(\Omega)$ and $v \in \mathcal{D}(\Omega)$. Then we have that

$$
a(u, v)=\int_{\Omega} A(t, x) u(x) v(x) \rho(x) \mathrm{d} x .
$$

2.1.3. The Gelfand triple. We can view $a^{0}$ as the principal term of the bilinear form $a(u, v)$. Let $\sigma$ denote the $n \times n_{\sigma}$ matrix whose $\sigma_{j}$ are the columns. Then

$$
a^{0}(u, v)=\sum_{i, j=1}^{n_{\sigma}} \int_{\Omega} \sigma_{j}[u] \sigma_{i}[v] \kappa_{i j} \rho=\int_{\Omega} \nabla u^{\top} \sigma \kappa \sigma^{\top} \nabla v \rho .
$$

Since $\kappa \succeq 0$, the above integrand is nonnegative when $u=v$; therefore, $a^{0}(u, u) \geq 0$. When $\kappa$ is the identity we have that $a^{0}(u, u)$ is equal to the seminorm $a^{00}(u, u)$, where

$$
a^{00}(u, u):=\int_{\Omega}\left|\sigma^{\top} \nabla u\right|^{2} \rho .
$$

In the presence of correlations it is natural to assume that we have a coercivity of the same order. That is, we assume that

$$
\text { For some } \gamma \in(0,1]: \quad \sigma \kappa \sigma^{\top} \succeq \gamma \sigma \sigma^{\top}, \quad \text { for all }(t, x) \in(0, T) \times \Omega \text {. }
$$

Therefore, we have

$$
a^{0}(u, u) \geq \gamma a^{00}(u, u)
$$

REMARK 2.2. Condition (2.18) holds in particular if

$$
\kappa \succeq \gamma I,
$$

but may also hold in other situations, e.g., when $n=1, n_{\sigma}=2, \kappa_{12}=1$, and $\sigma_{1}=\sigma_{2}=1$. Yet when the $\sigma_{i}$ are linearly independent, (2.19) is equivalent to (2.20). 
We need to choose a pair $(V, H)$ of Hilbert spaces satisfying the Gelfand conditions for the variational setting of Appendix A, namely $V$ densely and continuously embedded in $H, a(\cdot, \cdot)$ continuous and semi-coercive over $V$. Additionally, the r.h.s. and final condition of $(2.5)$ should belong to $L^{2}\left(0, T ; V^{*}\right)$ and $H$ resp. (and for the second parabolic estimate, to $L^{2}(0, T ; H)$ and $V$ resp. ).

We do as follows: for some measurable function $h: \Omega \rightarrow \mathbb{R}_{+}$to be specified later we define

$$
\left\{\begin{array}{l}
H:=\left\{v \in L^{0}(\Omega) ; \quad h v \in L^{2, \rho}(\Omega)\right\}, \\
\mathcal{V}:=\left\{v \in H ; \quad \sigma_{i}[v] \in L^{2, \rho}(\Omega), \quad i=1, \ldots, n_{\sigma}\right\}, \\
V:=\{\text { closure of } \mathcal{D}(\Omega) \text { in } \mathcal{V}\},
\end{array}\right.
$$

endowed with the natural norms,

$$
\|v\|_{H}:=\|h v\|_{\rho} ; \quad\|u\|_{V}^{2}:=a^{00}(u, u)+\|u\|_{H}^{2} .
$$

We do not try to characterize the space $V$ since this is problem dependent.

Obviously, $a^{0}(u, v)$ is a bilinear continuous form over $\mathcal{V}$. We next need to choose $h$ so that $a(u, v)$ is a bilinear and semi-coercive continuous form, and $u_{T} \in H$.

2.2. Continuity and semi-coercivity of the bilinear form over $\mathcal{V}$. We will see that the analysis of $a^{0}$ to $a^{2}$ is relatively easy. It is less obvious to analyze the term

$$
a^{34}(u, v):=a^{3}(u, v)+a^{4}(u, v) .
$$

Let $\bar{q}_{i j}(t, x) \in \mathbb{R}^{n}$ be the vector with $k$ th component equal to

$$
\bar{q}_{i j k}:=\kappa_{i j} \sigma_{i}\left[\sigma_{k j}\right] .
$$

Set

$$
\hat{q}:=\sum_{i, j=1}^{n_{\sigma}} \bar{q}_{i j}, \quad q:=\hat{q}-b .
$$

Then by (2.11)-(2.12), we have that

$$
a^{34}(u, v)=\int_{\Omega} q[u] v \rho .
$$

We next need to assume that it is possible to choose $\eta_{k}$ in $L^{0}((0, T) \times \Omega)$, for $k=1$ to $n_{\sigma}$, such that

$$
q=\sum_{k=1}^{n_{\sigma}} \eta_{k} \sigma_{k}
$$

Often the $n \times n_{\sigma}$ matrix $\sigma(t, x)$ has a.e. rank $n$. Then the above decomposition is possible. However, the choice for $\eta$ is not necessarily unique. We will see in examples how to do it. Consider the following hypotheses:

(2.28) $h_{\sigma} \leq c_{\sigma} h, \quad$ where $h_{\sigma}:=\sum_{i, j=1}^{n_{\sigma}}\left|\sigma_{i}\left[\kappa_{i j} \rho\right] / \rho+\kappa_{i j} \operatorname{div} \sigma_{i}\right|$, a.e., for some $c_{\sigma}>0$,

(2.29) $h_{r} \leq c_{r} h$, where $h_{r}:=|r|^{1 / 2}$, a.e., for some $c_{r}>0$,

(2.30) $h_{\eta} \leq c_{\eta} h$, where $h_{\eta}:=|\eta|$, a.e., for some $c_{\eta}>0$. 
REMARK 2.3. Let us set for any differentiable vector field $Z: \Omega \rightarrow \mathbb{R}^{n}$

$$
G_{\rho}(Z):=\operatorname{div} Z+\frac{Z[\rho]}{\rho} .
$$

Since $\kappa_{i i}=1,(2.28)$ implies that

$$
\left|G_{\rho}\left(\sigma_{i}\right)\right| \leq c_{\sigma} h, \quad i=1 ; \ldots, n_{\sigma} .
$$

REMARK 2.4. Since

$$
\sigma_{i}\left[\kappa_{i j} \rho\right]=\sigma_{i}\left[\kappa_{i j}\right] \rho+\sigma_{i}[\rho] \kappa_{i j},
$$

and $\left|\kappa_{i j}\right| \leq 1$ a.e., a sufficient condition for (2.28) is that there exist a positive constants $c_{\sigma}^{\prime}$ such that

$$
h_{\sigma}^{\prime} \leq c_{\sigma}^{\prime} h ; \quad h_{\sigma}^{\prime}:=\sum_{i, j=1}^{n_{\sigma}}\left|\sigma_{i}\left[\kappa_{i j}\right]\right|+\sum_{i=1}^{n_{\sigma}}\left(\left|\operatorname{div} \sigma_{i}\right|+\left|\sigma_{i}[\rho] / \rho\right|\right) .
$$

We will see in section 4 how to choose the weight $\rho$ so that $\left|\sigma_{i}[\rho] / \rho\right|$ can be easily estimated as a function of $\sigma$.

Lemma 2.5. Let (2.28)-(2.30) hold. Then the bilinear form $a(u, v)$ is both (i) continuous over $V$, and (ii) semi-coercive, in the sense of (A.5).

Proof. (i) We have that $a^{1}+a^{2}$ is continuous, since by (2.9)-(2.10), (2.28) and the Cauchy-Schwarz inequality:

$$
\begin{aligned}
\left|a^{1}(u, v)+a^{2}(u, v)\right| & \leq \sum_{i, j=1}^{n_{\sigma}}\left|a_{i j}^{1}(u, v)+a_{i j}^{2}(u, v)\right| \\
& \leq \sum_{j=1}^{n_{\sigma}}\left\|\sigma_{j}[u]\right\|_{\rho} \sum_{i=1}^{n_{\sigma}}\left\|\left(\sigma_{i}\left[\kappa_{i j} \rho\right] / \rho+\kappa_{i j} \operatorname{div} \sigma_{i}\right) v\right\|_{\rho} \\
& \leq c_{\sigma} n_{\sigma}\|v\|_{H} \sum_{j=1}^{n_{\sigma}}\left\|\sigma_{j}[u]\right\|_{\rho} .
\end{aligned}
$$

(ii) Also, $a^{34}$ is continuous, since by (2.27) and (2.30):

$$
\left|a^{34}(u, v)\right| \leq \sum_{k=1}^{n_{\sigma}}\left\|\sigma_{k}[u]\right\|_{\rho}\left\|\eta_{k} v\right\|_{\rho} \leq c_{\eta}\|v\|_{H} \sum_{k=1}^{n_{\sigma}}\left\|\sigma_{k}[u]\right\|_{\rho} .
$$

Set $c:=c_{\sigma} n_{\sigma}+c_{\eta}^{2}$. By (2.35)-(2.36), we have that

$$
\left\{\begin{array}{l}
\left|a^{5}(u, v)\right| \leq\left\||r|^{1 / 2} u\right\|_{2, \rho}\left\||r|^{1 / 2} v\right\|_{2, \rho} \leq c_{r}^{2}\|u\|_{H}\|v\|_{H}, \\
\left|a^{1}(u, v)+a^{2}(u, v)+a^{34}(u, v)\right| \leq c a^{00}(u)^{1 / 2}\|v\|_{H} .
\end{array}\right.
$$

Since $a^{0}$ is obviously continuous, the continuity of $a(u, v)$ follows.

(iii) Semi-coercivity. Using (2.37) and Young's inequality, we get that

$$
\begin{aligned}
a(u, u) & \geq a_{0}(u, u)-\left|a^{1}(u, u)+a^{2}(u, u)+a^{34}(u, u)\right|-\left|a^{5}(u, u)\right| \\
& \geq \gamma a^{00}(u)-c a^{00}(u)^{1 / 2}\|u\|_{H}-c_{r}\|u\|_{H}^{2} \\
& \geq \frac{1}{2} \gamma a^{00}(u)-\left(\frac{1}{2} \frac{c^{2}}{\gamma}+c_{r}\right)\|u\|_{H}^{2},
\end{aligned}
$$

which means that $a$ is semi-coercive. 
The above consideration allow to derive well-posedness results for parabolic equations and parabolic variational inequalities.

THEOREM 2.6. (i) Let $(V, H)$ be given by (2.21), with h satisfying (2.28)-(2.30), $\left(f, u_{T}\right) \in L^{2}\left(0, T ; V^{*}\right) \times H$. Then equation $(2.5)$ has a unique solution u in $L^{2}(0, T ; V)$ with $\dot{u} \in L^{2}\left(0, T ; V^{*}\right)$, and the mapping $\left(f, u_{T}\right) \mapsto u$ is nondecreasing. (ii) If in addition the semi-symmetry condition (A.8) holds, then $u$ in $L^{\infty}(0, T ; V)$ and $\dot{u} \in$ $L^{2}(0, T ; H)$.

Proof. This is a direct consequence of Propositions A.1, A.2 and C.1.

We next consider the case of parabolic variational inequalities associated with the set

$$
K:=\{\psi \in V: \psi(x) \geq \Psi(x) \text { a.e. in } \Omega\},
$$

where $\Psi \in V$. The strong and weak formulations of the parabolic variational inequality are defined in (B.2) and (B.5) resp. The abstract notion of monotonicity is discussed in appendix B. We denote by $\mathcal{K}$ the closure of $K$ in $V$.

ThEOREM 2.7. (i) Let the assumptions of theorem 2.6 hold, with $u_{T} \in \mathcal{K}$. Then the weak formulation (B.5) has a unique solution $u$ in $L^{2}(0, T ; K) \cap C(0, T ; H)$, and the mapping $\left(f, u_{T}\right) \mapsto u$ is nondecreasing.

(ii) Let in addition the semi-symmetry condition (A.8) be satisfied. Then $u$ is the unique solution of the strong formulation (B.2), belongs to $L^{\infty}(0, T ; V)$, and $\dot{u}$ belongs to $L^{2}(0, T ; H)$.

Proof. This follows from Propositions B.1 and C.2.

3. Variational analysis using the commutator analysis. In the following a commutator for first order differential operators is introduced, and calculus rules are derived.

3.1. Commutators. Let $u: \Omega \rightarrow \mathbb{R}$ be of class $C^{2}$. Let $\Phi$ and $\Psi$ be two vector fields over $\Omega$, both of class $C^{1}$. Recalling (1.11), we may define the commutator of the first-order differential operators associated with $\Phi$ and $\Psi$ as

$$
[\Phi, \Psi][u]:=\Phi[\Psi[u]]-\Psi[\Phi[u]]
$$

Note that

$$
\Phi[\Psi[u]]=\sum_{i=1}^{n} \Phi_{i} \frac{\partial(\Psi u)}{\partial x_{i}}=\sum_{i=1}^{n} \Phi_{i}\left(\sum_{k=1}^{n} \frac{\partial \Psi_{k}}{\partial x_{i}} \frac{\partial u}{\partial x_{k}}+\Psi_{k} \frac{\partial^{2} u}{\partial x_{k} \partial x_{i}}\right) .
$$

So, the expression of the commutator is

$$
\begin{aligned}
{[\Phi, \Psi][u] } & =\sum_{i=1}^{n}\left(\Phi_{i} \sum_{k=1}^{n} \frac{\partial \Psi_{k}}{\partial x_{i}} \frac{\partial u}{\partial x_{k}}-\Psi_{i} \sum_{k=1}^{n} \frac{\partial \Phi_{k}}{\partial x_{i}} \frac{\partial u}{\partial x_{k}}\right) \\
& =\sum_{k=1}^{n}\left(\sum_{i=1}^{n} \Phi_{i} \frac{\partial \Psi_{k}}{\partial x_{i}}-\Psi_{i} \frac{\partial \Phi_{k}}{\partial x_{i}}\right) \frac{\partial u}{\partial x_{k}} .
\end{aligned}
$$

It is another first-order differential operator associated with a vector field (which happens to be the Lie bracket of $\Phi$ and $\Psi$, see e.g.[4]). 
3.2. Adjoint. Remembering that $H$ was defined in (2.21), given two vector fields $\Phi$ and $\Psi$ over $\Omega$, we define the spaces

$$
\begin{aligned}
\mathcal{V}(\Phi, \Psi) & :=\{v \in H ; \quad \Phi[v], \Psi[v] \in H\} \\
V(\Phi, \Psi) & :=\{\text { closure of } \mathcal{D}(\Omega) \text { in } \mathcal{V}(\Phi, \Psi)\}
\end{aligned}
$$

We define the adjoint $\Phi^{\top}$ of $\Phi$ (view as an operator over say $C^{\infty}(\Omega, \mathbb{R})$, the latter being endowed with the scalar product of $L^{2, \rho}(\Omega)$ ), by

$$
\left\langle\Phi^{\top}[u], v\right\rangle_{\rho}=\langle u, \Phi[v]\rangle_{\rho} \quad \text { for all } u, v \in \mathcal{D}(\Omega),
$$

where $\langle\cdot, \cdot\rangle_{\rho}$ denotes the scalar product in $L^{2, \rho}(\Omega)$. Thus, there holds the identity

$$
\int_{\Omega} \Phi^{\top}[u](x) v(x) \rho(x) \mathrm{d} x=\int_{\Omega} u(x) \Phi[v](x) \rho(x) \mathrm{d} x \quad \text { for all } u, v \in \mathcal{D}(\Omega) .
$$

Furthermore,

$$
\begin{aligned}
\int_{\Omega} u \sum_{i=1}^{n} \Phi_{i} \frac{\partial v}{\partial x_{i}} \rho \mathrm{d} x & =-\sum_{i=1}^{n} \int_{\Omega} v \frac{\partial}{\partial x_{i}}\left(u \rho \Phi_{i}\right) \mathrm{d} x \\
& =-\sum_{i=1}^{n} \int_{\Omega} v\left(\frac{\partial}{\partial x_{i}}\left(u \Phi_{i}\right)+\frac{u}{\rho} \Phi_{i} \frac{\partial \rho}{\partial x_{i}}\right) \rho \mathrm{d} x .
\end{aligned}
$$

Hence,

$$
\Phi^{\top}[u]=-\sum_{i=1}^{n} \frac{\partial}{\partial x_{i}}\left(u \Phi_{i}\right)-u \Phi_{i} \frac{\partial \rho}{\partial x_{i}} / \rho=-u \operatorname{div} \Phi-\Phi[u]-u \Phi[\rho] / \rho .
$$

Remembering the definition of $G_{\rho}(\Phi)$ in (2.31), we obtain that

$$
\Phi[u]+\Phi^{\top}[u]+G_{\rho}(\Phi) u=0 .
$$

3.3. Continuity of the bilinear form associated with the commutator. Setting, for $v$ and $w$ in $V(\Phi, \Psi)$ :

$$
\Delta(u, v):=\int_{\Omega}[\Phi, \Psi][u](x) v(x) \rho(x) \mathrm{d} x,
$$

we have

$$
\begin{aligned}
\Delta(u, v) & \left.=\int_{\Omega}(\Phi[\Psi[u]] v-\Psi[\Phi[u]] v) \rho \mathrm{d} x=\int_{\Omega} \Psi[u] \Phi^{\top}[v]-\Phi[u] \Psi^{\top}[v]\right) \rho \mathrm{d} x \\
& =\int_{\Omega}(\Phi[u] \Psi[v]-\Psi[u] \Phi[v]) \rho \mathrm{d} x+\int_{\Omega}\left(\Phi[u] G_{\rho}(\Psi) v-\Psi[u] G_{\rho}(\Phi) v\right) \rho \mathrm{d} x .
\end{aligned}
$$

Lemma 3.1. For $\Delta(\cdot, \cdot)$ to be a continuous bilinear form on $V(\Phi, \Psi)$, it suffices that, for some $c_{\Delta}>0$ :

$$
\left|G_{\rho}(\Phi)\right|+\left|G_{\rho}(\Psi)\right| \leq c_{\Delta} h \quad \text { a.e. }
$$

and we have then:

$$
|\Delta(u, v)| \leq\|\Psi[u]\|_{\rho}\left(\|\Phi[v]\|_{\rho}+c_{\Delta}\|v\|_{H}\right)+\|\Phi[u]\|_{\rho}\left(\|\Psi[v]\|_{\rho}+c_{\Delta}\|v\|_{H}\right) .
$$


Proof. Apply the Cauchy Schwarz inequality to (3.12), and use (3.13) combined with the definition of the space $H$.

We apply the previous results with $\Phi:=\sigma_{i}, \Psi:=\sigma_{j}$. Set for $v, w$ in $V$ :

$$
\Delta_{i j}(u, v):=\int_{\Omega}\left[\sigma_{i}, \sigma_{j}\right][u](x) v(x) \rho(x) \mathrm{d} x, \quad i, j=1, \ldots, n_{\sigma} .
$$

We recall that $V$ was defined in (2.21).

Corollary 3.2. Let (2.28) hold. Then the $\Delta_{i j}(u, v), i, j=1, \ldots, n_{\sigma}$, are continuous bilinear forms over $V$.

Proof. Use remark 2.3 and conclude with lemma 3.1.

3.4. Redefining the space $H$. In section 2.2 we have obtained the continuity and semi-coercivity of $a$ by decomposing $q$, defined in (2.26), as a linear combination (2.27) of the $\sigma_{i}$. We now take advantage of the previous computation of commutators and assume that, more generally, instead of (2.27), we can decompose $q$ in the form

$$
q=\sum_{k=1}^{n_{\sigma}} \eta_{k}^{\prime \prime} \sigma_{k}+\sum_{1 \leq i<j \leq n_{\sigma}} \eta_{i j}^{\prime}\left[\sigma_{i}, \sigma_{j}\right] \quad \text { a.e. }
$$

We assume that $\eta^{\prime}$ and $\eta^{\prime \prime}$ are measurable functions over $[0, T] \times \Omega$, that $\eta^{\prime}$ is weakly differentiable, and that for some $c_{\eta}^{\prime}>0$ :

$$
h_{\eta}^{\prime} \leq c_{\eta}^{\prime} h, \text { where } h_{\eta}^{\prime}:=\left|\eta^{\prime \prime}\right|+\sum_{i, j=1}^{N}\left|\sigma_{i}\left[\eta_{i j}^{\prime}\right]\right| \quad \text { a.e., } \eta^{\prime} \in L^{\infty}(\Omega) .
$$

Lemma 3.3. Let (2.28), (2.29), and (3.17) hold. Then the bilinear form a $(u, v)$ defined in (2.14) is both (i) continuous and (ii) semi-coercive over $V$.

Proof. (i) We only have to analyze the contribution of $a^{34}$ (defined in (2.23)), since the other contributions to $a(\cdot, \cdot)$ do not change. For the terms in the first sum in (3.16) we have, as was done in (2.36):

$$
\left|\int_{\Omega} \sigma_{k}[u] \eta_{k}^{\prime \prime} v \rho\right| \leq\left\|\sigma_{k}[u]\right\|_{\rho}\left\|\sigma_{k}[u] \eta_{k}^{\prime \prime} v\right\|_{\rho} \leq\left\|\sigma_{k}[u]\right\|_{\rho}\|v\|_{H} .
$$

(ii) Setting $w:=\eta_{i j}^{\prime} v$ and taking here $(\Phi, \Psi)=\left(\sigma_{i}, \sigma_{j}\right)$, we get that

$$
\int_{\Omega} \eta_{i j}^{\prime}\left[\sigma_{i}, \sigma_{j}\right)[u] v \rho=\Delta(u, w),
$$

where $\Delta(\cdot, \cdot)$ was defined in (3.11). Combining with lemma 3.1, we obtain

$$
\begin{aligned}
\left|\Delta_{i j}(u, v)\right| \leq & \left\|\sigma_{j}[u]\right\|_{\rho}\left(\left\|\sigma_{i}[w]\right\|_{\rho}+c_{\sigma}\left\|\eta_{i j}^{\prime}\right\|_{\infty}\|v\|_{H}\right) \\
& +\left\|\sigma_{i}[u]\right\|_{\rho}\left(\left\|\sigma_{j}[w]\right\|_{\rho}+c_{\sigma}\left\|\eta_{i j}^{\prime}\right\|_{\infty}\|v\|_{H}\right) .
\end{aligned}
$$

Since

$$
\sigma_{i}\left[\eta_{i j}^{\prime} v\right]=\eta_{i j}^{\prime} \sigma_{i}[v]+\sigma_{i}\left[\eta_{i j}^{\prime}\right] v
$$


by (3.17):

$$
\left\|\sigma_{i}[w]\right\|_{\rho} \leq\left\|\eta_{i j}^{\prime}\right\|_{\infty}\left\|\sigma_{i}[v]\right\|_{\rho}+\left\|\sigma_{i}\left[\eta_{i j}^{\prime}\right] v\right\|_{\rho} \leq\left\|\eta_{i j}^{\prime}\right\|_{\infty}\left\|\sigma_{i}[v]\right\|_{\rho}+c_{\eta}\|v\|_{H} .
$$

Combining these inequalities, point (i) follows.

(ii) Use $u=v$ in (3.21) and (3.12). We find after cancellation in (3.12) that

$$
\begin{aligned}
\Delta_{i j}\left(u, \eta_{i j}^{\prime} u\right)= & \int_{\Omega} u\left(\sigma_{i}[u] \sigma_{j}\left[\eta_{i j}^{\prime}\right]-\sigma_{j}[u] \sigma_{i}\left(\eta_{i j}^{\prime}\right)\right) \rho \\
& +\int_{\Omega}\left(\sigma_{i}[u] G_{\rho}\left(\sigma_{j}\right)-\sigma_{j}[u] G_{\rho}\left(\sigma_{i}\right)\right) \eta_{i j}^{\prime} u \rho .
\end{aligned}
$$

By (3.17), an upper bound for the absolute value of the first integral is

$$
\left(\left\|\sigma_{i}[u]\right\|_{\rho}+\left\|\sigma_{j}[u]\right\|_{\rho}\right)\|h u\|_{\rho} \leq 2\|u\|_{\mathcal{V}}\|u\|_{H} .
$$

With (2.28), we get an upper bound for the absolute value of the second integral in the same way, so, for any $\varepsilon>0$ :

$$
\left|\Delta_{i j}\left(u, \eta_{i j}^{\prime} u\right)\right| \leq 4\|u\|_{\mathcal{V}}\|u\|_{H} .
$$

We finally have that for some $c>0$

$$
\begin{aligned}
a(u, u) & \geq a_{0}(u, u)-c\|u\|_{\mathcal{V}}\|u\|_{H}, \\
& \geq a_{0}(u, u)-\frac{1}{2}\|u\|_{\mathcal{V}}^{2}-\frac{1}{2} c^{2}\|u\|_{H}^{2}, \\
& =\frac{1}{2}\|u\|_{\mathcal{V}}^{2}-\frac{1}{2}\left(c^{2}+1\right)\|u\|_{H}^{2} .
\end{aligned}
$$

The conclusion follows.

REMARK 3.4. The statements analogous to theorems 2.6 and 2.7 hold, assuming now that $h$ satisfies (2.28), (2.29), and (3.17) (instead of (2.28)-(2.30)).

4. The weight $\rho$. Classes of weighting functions characterized by their growth are introduced. A major result is the independence of the growth order of the function $h$ on the choice of the weighting function $\rho$ in the class under consideration.

4.1. Classes of functions with given growth. In financial models we usually have nonnegative variables and the related functions have polynomial growth. Yet, after a logarithmic transformation, we get real variables whose related functions have exponential growth. This motivates the following definitions.

We remind that $(I, J)$ is a partition of $\{0, \ldots, N\}$, with $0 \in J$ and that $\Omega$ was defined in (1.8).

Definition 4.1. Let $\gamma^{\prime}$ and $\gamma^{\prime \prime}$ belong to $\mathbb{R}_{+}^{N+1}$, with index from 0 to $N$. Let $\mathcal{G}\left(\gamma^{\prime}, \gamma^{\prime \prime}\right)$ be the class of functions $\varphi: \Omega \rightarrow \mathbb{R}$ such that for some $c>0$ :

$$
|\varphi(x)| \leq c\left(\prod_{k \in I}\left(e^{\gamma_{k}^{\prime} x_{k}}+e^{-\gamma_{k}^{\prime \prime} x_{k}}\right)\right)\left(\prod_{k \in J}\left(x_{k}^{\gamma_{k}^{\prime}}+x_{k}^{-\gamma_{k}^{\prime \prime}}\right)\right) .
$$

We define $\mathcal{G}$ as the union of $\mathcal{G}\left(\gamma^{\prime}, \gamma^{\prime \prime}\right)$ for all nonnegative $\left(\gamma^{\prime}, \gamma^{\prime \prime}\right)$. We call $\gamma_{k}^{\prime}$ and $\gamma_{k}^{\prime \prime}$ the growth order of $\varphi$, w.r.t. $x_{k}$, at $-\infty$ and $+\infty$ (resp. at zero and $+\infty$ ).

Observe that the class $\mathcal{G}$ is stable by the operations of sum and product, and that if $f, g$ belong to that class, so does $h=f g, h$ having growth orders equal to the sum of the growth orders of $f$ and $g$. For $a \in \mathbb{R}$, we define

$$
a^{+}:=\max (0, a) ; \quad a^{-}:=\max (0,-a) ; \quad N(a):=\left(a^{2}+1\right)^{1 / 2},
$$


as well as

$$
\rho:=\rho_{I} \rho_{J}
$$

where

$$
\begin{aligned}
& \rho_{I}(x):=\prod_{k \in I} e^{-\alpha_{k}^{\prime} N\left(x_{k}^{+}\right)-\alpha_{k}^{\prime \prime} N\left(x_{k}^{-}\right)}, \\
& \rho_{J}(x):=\prod_{k \in J} \frac{x_{k}^{\alpha_{k}^{\prime}}}{1+x_{k}^{\alpha_{k}^{\prime}+\alpha_{k}^{\prime \prime}}},
\end{aligned}
$$

for some nonnegative constants $\alpha_{k}^{\prime}, \alpha_{k}^{\prime \prime}$, to be specified later.

LEMma 4.2. Let $\varphi \in \mathcal{G}\left(\gamma^{\prime}, \gamma^{\prime \prime}\right)$. Then $\varphi \in L^{1, \rho}(\Omega)$ whenever $\rho$ is as above, with $\alpha$ satisfying, for some positive $\varepsilon^{\prime}$ and $\varepsilon^{\prime \prime}$, for all $k=0$ to $N$ :

$$
\left\{\begin{array}{lll}
\alpha_{k}^{\prime}=\varepsilon^{\prime}+\gamma_{k}^{\prime}, & \alpha_{k}^{\prime \prime}=\varepsilon^{\prime \prime}+\gamma_{k}^{\prime \prime}, & k \in I, \\
\alpha_{k}^{\prime}=\left(\varepsilon^{\prime}+\gamma_{k}^{\prime \prime}-1\right)_{+}, & \alpha_{k}^{\prime \prime}=1+\varepsilon^{\prime \prime}+\gamma_{k}^{\prime}, & k \in J .
\end{array}\right.
$$

In addition we can choose for $k=0$ (if element of $J$ ):

$$
\left\{\begin{array}{l}
\alpha_{0}^{\prime}:=\left(\varepsilon^{\prime}+\gamma_{0}^{\prime \prime}-1\right)_{+} ; \alpha_{0}^{\prime \prime}:=0 \quad \text { if } \varphi(s, y)=0 \text { when } s \text { is far from } 0, \\
\alpha_{0}^{\prime}:=0, \alpha_{0}^{\prime \prime}:=1+\varepsilon^{\prime \prime}+\gamma_{0}^{\prime}, \quad \text { if } \varphi(s, y)=0 \text { when } s \text { is close to } 0 .
\end{array}\right.
$$

Proof. It is enough to prove (4.6), the proof of (4.7) is similar. We know that $\varphi$ satisfy (4.1) for some $c>0$ and $\gamma$. We need to check the finiteness of

$$
\int_{\Omega}\left(\prod_{k \in I}\left(e^{\gamma_{k}^{\prime} y_{k}}+e^{-\gamma_{k}^{\prime \prime} y_{k}}\right)\right)\left(\prod_{k \in J}\left(y_{k}^{\gamma_{k}^{\prime}}+y_{k}^{-\gamma_{k}^{\prime \prime}}\right)\right) \rho(s, y) \mathrm{d}(s, y) .
$$

But the above integral is equal to the product $p_{I} p_{J}$ with

$$
\begin{aligned}
p_{I} & :=\prod_{k \in I} \int_{\mathbb{R}}\left(e^{\gamma_{k}^{\prime} x_{k}}+e^{-\gamma_{k}^{\prime \prime} x_{k}}\right) e^{-\alpha_{k}^{\prime} N\left(x_{k}^{+}\right)-\alpha_{k}^{\prime \prime} N\left(x_{k}^{-}\right)} \mathrm{d} x_{k}, \\
p_{J} & :=\prod_{k \in J} \int_{\mathbb{R}_{+}} \frac{x_{k}^{\alpha_{k}^{\prime}+\gamma_{k}^{\prime}}+x_{k}^{\alpha_{k}^{\prime}-\gamma_{k}^{\prime \prime}}}{1+x_{k}^{\alpha_{k}^{\prime}+\alpha_{k}^{\prime \prime}}} \mathrm{d} x_{k} .
\end{aligned}
$$

Using (4.6) we deduce that $p_{I}$ is finite since for instance

$$
\begin{aligned}
& \int_{\mathbb{R}_{+}}\left(e^{\gamma_{k}^{\prime} x_{k}}+e^{-\gamma_{k}^{\prime \prime} x_{k}}\right) e^{-\alpha_{k}^{\prime} N\left(x_{k}^{+}\right)-\alpha_{k}^{\prime \prime} N\left(x_{k}^{-}\right)} \mathrm{d} x_{k} \\
& \leq 2 \int_{\mathbb{R}_{+}} e^{\gamma_{k}^{\prime} x_{k}} e^{-\left(1+\gamma_{k}^{\prime}\right) x_{k}} \mathrm{~d} x_{k}=2 \int_{\mathbb{R}_{+}} e^{-x_{k}} \mathrm{~d} x_{k}=2,
\end{aligned}
$$

and $p_{J}$ is finite since

$$
p_{J}=\prod_{k \in J} \int_{\mathbb{R}_{+}} \frac{x_{k}^{\varepsilon^{\prime}+\gamma_{k}^{\prime}+\gamma_{k}^{\prime \prime}}+x_{k}^{\varepsilon^{\prime}-1}}{1+x_{k}^{\varepsilon^{\prime}+\varepsilon^{\prime \prime}+\gamma_{k}^{\prime}+\gamma_{k}^{\prime \prime}}} \mathrm{d} x_{k}<\infty .
$$

The conclusion follows. 


\subsection{On the growth order of $h$. Set for all $k$}

$$
\alpha_{k}:=\alpha_{k}^{\prime}+\alpha_{k}^{\prime \prime} .
$$

Remember that we take $\rho$ in the form (4.3)-(4.4).

LEMMA 4.3. We have that:

(i) We have that

$$
\left\|\frac{\rho_{x_{k}}}{\rho}\right\|_{\infty} \leq \alpha_{k}, \quad k \in I ; \quad\left\|\frac{x}{\rho} \rho_{x_{k}}\right\|_{\infty} \leq \alpha_{k}, \quad k \in J .
$$

(ii) Let h satisfying either (2.28)-(2.30) or (2.28)-(2.29), and (3.17). Then the growth order of $h$ does not depend on the choice of the weighting function $\rho$.

Proof. (i) For $k \in I$ this is an easy consequence of the fact that $N(\cdot)$ is non expansive. For $k \in J$, we have that

$$
\frac{x}{\rho} \rho_{x_{k}}=\frac{x}{\rho} \frac{\alpha_{k}^{\prime} x^{\alpha_{k}^{\prime}-1}\left(1+x^{\alpha_{k}}\right)-x^{\alpha_{k}^{\prime}} \alpha_{k} x^{\alpha_{k}-1}}{\left(1+x^{\alpha_{k}}\right)^{2}}=\frac{\alpha_{k}^{\prime}-\alpha_{k}^{\prime \prime} x^{\alpha_{k}}}{1+x^{\alpha_{k}}} .
$$

We easily conclude, discussing the sign of the numerator.

(ii) The dependence of $h$ w.r.t. $\rho$ is only through the last term in (2.28), namely, $\sum_{i} \mid \sigma_{i}[\rho] / \rho$. By (i) we have that

$$
\left|\frac{\sigma_{i}^{k}[\rho]}{\rho}\right| \leq\left\|\frac{\rho_{x_{k}}}{\rho}\right\|_{\infty}\left|\sigma_{i}^{k}\right| \leq \alpha_{k}\left|\sigma_{i}^{k}\right|, \quad k \in I
$$

$$
\left|\frac{\sigma_{i}^{k}[\rho]}{\rho}\right| \leq\left\|\frac{x_{k} \rho_{x_{k}}}{\rho}\right\|_{\infty}\left|\frac{\sigma_{i}^{k}}{x_{k}}\right| \leq \alpha_{k}\left|\frac{\sigma_{i}^{k}}{x_{k}}\right|, \quad k \in J
$$

In both cases, the choice of $\alpha$ has no influence on the growth order of $h$.

4.3. European option. In the case of a European option with payoff $u_{T}(x)$, we need to check that $u_{T} \in H$, that is, $\rho$ must satisfy

$$
\int_{\Omega}\left|u_{T}(x)\right|^{2} h(x)^{2} \rho(x) \mathrm{d} x<\infty .
$$

In the framework of the semi-symmetry hypothesis (A.8), we need to check that $u_{T} \in V$, which gives the additional condition

$$
\sum_{i=1}^{n_{\sigma}} \int_{\Omega}\left|\sigma_{i}\left[u_{T}\right](x)\right|^{2} \rho(x) \mathrm{d} x<\infty .
$$

In practice the payoff depends only on $s$ and this allows to simplify the analysis.

5. Applications using the commutator analysis. The commutator analysis is applied to the general multiple factor model and estimates for the function $h$ characterizing the space $H$ (defined in (2.21)) are derived. The estimates are compared to the case when the commutator analysis is not applied. The resulting improvement wil be established in the next section. 
5.1. Commutator and continuity analysis. We analyze the general multiple factor model (1.4), which belongs to the class of models (2.1) with $\Omega \subset \mathbb{R}^{1+N}, n_{\sigma}=$ $2 N$, and for $i=1$ to $N$ :

$$
\sigma_{i}[v]=f_{i}\left(y_{i}\right) s^{\beta_{i}} v_{s} ; \quad \sigma_{N+i}[v]=g_{i}\left(y_{i}\right) v_{i},
$$

with $f_{i}$ and $g_{i}$ of class $C^{1}$ over $\Omega$. We need to compute the commutators of the firstorder differential operators associated with the $\sigma_{i}$. The correlations will be denoted by

$$
\hat{\kappa}_{k}:=\kappa_{k, N+k}, \quad k=1, \ldots, N .
$$

REMARK 5.1. We use many times the following rule. For $\Omega \subset \mathbb{R}^{n}$, where $n=$ $1+N, u \in H^{1}(\Omega), a, b \in L^{0}$, and vector fields $Z[u]:=a u_{x_{1}}$ and $Z^{\prime}[u]:=b u_{x_{2}}$, we have $Z\left[Z^{\prime}[u]\right]=a\left(b u_{x_{2}}\right)_{x_{1}}=a b_{x_{1}} u_{x_{2}}+a b u_{x_{1} x_{2}}$, so that

$$
\left[Z, Z^{\prime}\right][u]=a b_{x_{1}} u_{x_{2}}-b a_{x_{2}} u_{x_{1}} .
$$

We obtain that

$$
\left[\sigma_{i}, \sigma_{N+i}\right][u]=-s^{\beta_{i}} f_{i}^{\prime}\left(y_{i}\right) g_{i}\left(y_{i}\right) u_{s}, \quad i=1, \ldots, N,
$$

and

$$
\left[\sigma_{i}, \sigma_{N+\ell}\right][u]=\left[\sigma_{N+i}, \sigma_{N+\ell}\right][u]=0, \quad i \neq \ell .
$$

Also,

$$
\begin{array}{ll}
\operatorname{div} \sigma_{i}+\frac{\sigma_{i}[\rho]}{\rho} & =f_{i}\left(y_{i}\right) s^{\beta_{i}-1}\left(\beta_{i}+s \frac{\rho_{s}}{\rho}\right), \\
\operatorname{div} \sigma_{N+i}+\frac{\sigma_{N+i}[\rho]}{\rho} & =g_{i}^{\prime}\left(y_{i}\right)+g_{i}\left(y_{i}\right) \frac{\rho_{i}}{\rho} .
\end{array}
$$

5.1.1. Computation of $q$. Remember the definitions of $\bar{q}, \hat{q}$ and $q$ in $(2.24)$ and (2.25), where $\delta_{i j}$ denote the Kronecker operator. We obtain that, for $1 \leq i, j, k \leq N$ :

$$
\left\{\begin{array}{lll}
\bar{q}_{i j 0} & =\delta_{i j} \beta_{j} f_{i}^{2}\left(y_{i}\right) s^{2 \beta_{i}-1} ; \quad \bar{q}_{i i k}=0 ; \\
\bar{q}_{i, N+j} & =0 ; \\
\bar{q}_{N+i, j, 0} & =\delta_{i j} \hat{\kappa}_{i} f^{\prime}\left(y_{i}\right) g_{i}\left(y_{i}\right) s^{\beta_{i}} ; \quad \bar{q}_{N+i, j, k}=0 ; \\
\bar{q}_{N+i, N+j, k} & =\delta_{i j k} g_{i}\left(y_{i}\right) g_{i}^{\prime}\left(y_{i}\right) .
\end{array}\right.
$$

That means, we have for $\hat{q}=\sum_{i, j=1}^{2 N} \bar{q}_{i j}$ and $q=\hat{q}-b$ that

$$
\begin{array}{ll}
\hat{q}_{0}=\sum_{i=1}^{N}\left(\beta_{i} f_{i}^{2}\left(y_{i}\right) s^{2 \beta_{i}-1}+\hat{\kappa}_{i} f^{\prime}\left(y_{i}\right) g_{i}\left(y_{i}\right) s^{\beta_{i}}\right) ; & q_{0}=\hat{q}_{0}-r s, \\
& q_{k}=\hat{q}_{k}-\theta_{k}\left(\mu_{k}-y_{k}\right), \\
& k=1, \ldots, N
\end{array}
$$


5.2. Computation of $\eta^{\prime}$ and $\eta^{\prime \prime}$. The coefficients $\eta^{\prime}, \eta^{\prime \prime}$ are solution of (3.16). We can write $\eta=\hat{\eta}+\tilde{\eta}$, where

$$
\begin{aligned}
\hat{q} & =\sum_{i=1}^{n_{\sigma}} \hat{\eta}_{i}^{\prime \prime} \sigma_{i}+\sum_{1 \leq i, j \leq n_{\sigma}} \hat{\eta}_{i j}^{\prime}\left[\sigma_{i}, \sigma_{j}\right], \quad \hat{\eta}_{i j}^{\prime}=0 \text { if } i=j . \\
-b & =\sum_{i=1}^{n_{\sigma}} \tilde{\eta}_{i}^{\prime \prime} \sigma_{i}+\sum_{1 \leq i, j \leq n_{\sigma}} \tilde{\eta}_{i j}^{\prime}\left[\sigma_{i}, \sigma_{j}\right], \quad \tilde{\eta}_{i j}^{\prime}=0 \text { if } i=j .
\end{aligned}
$$

For $k=1$ to $N$, this reduces to

$$
\left\{\begin{array}{l}
\hat{\eta}_{N+k}^{\prime \prime} g_{k}\left(y_{k}\right)=g_{k}^{\prime}\left(y_{k}\right) g_{k}\left(y_{k}\right) \\
\tilde{\eta}_{N+k}^{\prime \prime} g_{k}\left(y_{k}\right)=-\theta_{k}\left(\mu_{k}-y_{k}\right) .
\end{array}\right.
$$

So, we have that

$$
\left\{\begin{array}{l}
\hat{\eta}_{N+k}^{\prime \prime}=g_{k}^{\prime}\left(y_{k}\right) ; \\
\tilde{\eta}_{N+k}^{\prime \prime}=\frac{-\theta_{k}\left(\mu_{k}-y_{k}\right)}{g_{k}\left(y_{k}\right)} .
\end{array}\right.
$$

For the 0th component, (5.10) can be expressed as

$$
\left\{\begin{array}{l}
\sum_{k=1}^{N}\left(-\hat{\eta}_{k, N+k}^{\prime} f_{k}^{\prime}\left(y_{k}\right) g_{k}\left(y_{k}\right) s^{\beta_{k}}-\hat{\kappa}_{k} f_{k}^{\prime}\left(y_{k}\right) g_{k}\left(y_{k}\right) s^{\beta_{k}}\right) \\
+\sum_{k=1}^{N}\left(\hat{\eta}_{k}^{\prime \prime} f_{k}\left(y_{k}\right) s^{\beta_{k}}-\beta_{k} f_{k}^{2}\left(y_{k}\right) s^{2 \beta_{k}-1}\right) \\
+\sum_{k=1}^{N}\left(-\tilde{\eta}_{k, N+k}^{\prime} f_{k}^{\prime}\left(y_{k}\right) g_{k}\left(y_{k}\right) s^{\beta_{k}}+\tilde{\eta}_{k}^{\prime \prime} f_{k}\left(y_{k}\right) s^{\beta_{k}}\right)-r s=0 .
\end{array}\right.
$$

We choose to set each term in parenthesis in the first two lines above to zero. It follows that

$$
\hat{\eta}_{k, N+k}^{\prime}=-\hat{\kappa}_{k} \in L^{\infty}(\Omega), \quad \hat{\eta}_{k}^{\prime \prime}=\beta_{k} f_{k}\left(y_{k}\right) s^{\beta_{k}-1} .
$$

If $N>1$ we (arbitrarily) choose then to set the last line to zero with

$$
\tilde{\eta}_{k}^{\prime \prime}=\tilde{\eta}_{k}^{\prime}=0, \quad k=2, \ldots, N
$$

It remains that

$$
\tilde{\eta}_{1}^{\prime \prime} f_{1}\left(y_{1}\right) s^{\beta_{1}}-\tilde{\eta}_{1, N+1}^{\prime} f_{1}^{\prime}\left(y_{1}\right) g_{1}\left(y_{1}\right) s^{\beta_{1}}=r s .
$$

Here, we can choose to take either $\tilde{\eta}_{1}^{\prime \prime}=0$ or $\tilde{\eta}_{1, N+1}^{\prime}=0$. We obtain then two possibilities:

$$
\left\{\begin{array}{l}
\text { (i) } \quad \tilde{\eta}_{1}^{\prime \prime}=0 \text { and } \tilde{\eta}_{1, N+1}^{\prime}=\frac{-r s^{1-\beta_{1}}}{f_{1}^{\prime}\left(y_{1}\right) g_{1}\left(y_{1}\right)}, \\
\text { (ii) } \quad \tilde{\eta}_{1}^{\prime \prime}=\frac{r s^{1-\beta_{1}}}{f_{1}\left(y_{1}\right)} \text { and } \tilde{\eta}_{1, N+1}^{\prime}=0 .
\end{array}\right.
$$


5.2.1. Estimate of the $h$ function. We decide to choose case (i) in (5.17). The function $h$ needs to satisfy (2.28), (2.29), and (3.17) (instead of (2.30)). Instead of (2.28), we will rather check the stronger condition (2.34). We compute

$$
\begin{aligned}
h_{\sigma}^{\prime}:= & \sum_{k=1}^{N}\left|f_{k}\left(y_{k}\right)\right| s^{\beta_{k}}\left(\left|\left(\hat{\kappa}_{k}\right)_{s}\right|+\left|\frac{\rho_{s}}{\rho}\right|\right)+\left|g_{k}\left(y_{k}\right)\right|\left(\left|\left(\hat{\kappa}_{k}\right)_{k}\right|+\left|\frac{\rho_{k}}{\rho}\right|\right) \\
& +\sum_{k=1}^{N}\left(\beta_{k}\left|f_{k}\left(y_{k}\right) s^{\beta_{k}-1}\right|+\left|g_{k}^{\prime}\left(y_{k}\right)\right|\right) \\
h_{r}:= & |r|^{\frac{1}{2}} \\
h_{\eta}^{\prime}:= & \hat{h}_{\eta}^{\prime}+\tilde{h}_{\eta}^{\prime},
\end{aligned}
$$

where we have

$$
\hat{h}_{\eta}^{\prime}:=\sum_{k=1}^{N}\left(\beta_{k}\left|f_{k}\left(y_{k}\right)\right| s^{\beta_{k}-1}+\left|g_{k}^{\prime}\left(y_{k}\right)\right|+\left|f_{k}\left(y_{k}\right)\right| s^{\beta_{k}} \frac{\partial \hat{\kappa}_{k}}{\partial s}|+| g_{k}\left(y_{k}\right) \frac{\partial \hat{\kappa}_{k}}{\partial y_{k}} \mid\right),
$$

$$
\tilde{h}_{\eta}^{\prime}:=\sum_{k=1}^{N}\left|\frac{\theta_{k}\left(\mu_{k}-y_{k}\right)}{g_{k}\left(y_{k}\right)}\right|+\left|r \frac{f_{1}\left(y_{1}\right)}{f_{1}^{\prime}\left(y_{1}\right) g_{1}\left(y_{1}\right)}\right|+\left|r g_{1}\left(y_{1}\right) s^{1-\beta_{1}} \frac{\partial}{\partial y_{1}}\left[\frac{1}{f_{1}^{\prime}\left(y_{1}\right) g_{1}\left(y_{1}\right)}\right]\right| \text {. }
$$

REMARK 5.2. Had we chosen (ii) instead of (i) in (5.17), this would only change the expression of $\tilde{h}_{\eta}^{\prime}$ that would then be

$$
\tilde{h}_{\eta}^{\prime}=\sum_{k=1}^{N}\left|\frac{\theta_{k}\left(\mu_{k}-y_{k}\right)}{g_{k}\left(y_{k}\right)}\right|+\left|\frac{r s^{1-\beta_{1}}}{f_{1}\left(y_{1}\right)}\right| .
$$

5.2.2. Estimate of the $h$ function without the commutator analysis. The only change in the estimate of $h$ will be the contribution of $h_{\eta}^{\prime}$ and $h_{\eta}^{\prime \prime}$. We have to satisfy (2.28)-(2.30). In addition, ignoring the commutator analysis, we would solve (5.13) with $\hat{\eta}^{\prime}=0$, meaning that we choose

$$
\hat{\eta}_{k}^{\prime \prime}:=\beta_{k} f_{k}\left(y_{k}\right) s^{\beta_{k}-1}+\hat{\kappa}_{k} \frac{f_{k}^{\prime}\left(y_{k}\right) g_{k}\left(y_{k}\right)}{f_{k}\left(y_{k}\right)}, k=1, \ldots, N,
$$

and take $\tilde{\eta}_{1}^{\prime \prime}$ out of (5.16). Then condition (3.17), with here $\hat{\eta}^{\prime}=0$, would give

$$
h \geq c_{\eta} h_{\eta}, \text { where } h_{\eta}:=h_{\hat{\eta}}+h_{\tilde{\eta}},
$$

with

$$
\begin{aligned}
h_{\hat{\eta}} & :=\sum_{k=1}^{N}\left(\beta_{k}\left|f_{k}\left(y_{k}\right)\right| s^{\beta_{k}-1}+\left|\hat{\kappa}_{k}\right|\left|\frac{f_{k}^{\prime}\left(y_{k}\right) g_{k}\left(y_{k}\right)}{f_{k}\left(y_{k}\right)}\right|+\left|g_{k}^{\prime}\left(y_{k}\right)\right|\right), \\
h_{\tilde{\eta}} & :=\sum_{k=1}^{N}\left|\frac{\theta_{k}\left(\mu_{k}-y_{k}\right)}{g_{k}\left(y_{k}\right)}\right|+\left|\frac{r s^{1-\beta_{1}}}{f_{1}\left(y_{1}\right)}\right| .
\end{aligned}
$$

We will see in applications that this is in general worse. 
6. Application to stochastic volatility models. The results of Section 5 are specified for a subclass of the multiple factor model, in particular for the VAT and GMH models. We show that the commutator analysis allows to take smaller values for the function $h$ (and consequently to include a larger class of payoff functions).

6.1. A useful subclass. Here we assume that

$$
\left|f_{k}\left(y_{k}\right)\right|=\left|y_{k}\right|^{\gamma_{k}} ;\left|g_{k}\left(y_{k}\right)\right|=\nu_{k}\left|y_{k}\right|^{1-\gamma_{k}} ; \quad \beta_{k} \in(0,1] ; \quad \nu_{k}>0 ; \quad \gamma_{k} \in(0, \infty) .
$$

Furthermore, we assume $\kappa$ to be constant and

$$
\left|f_{k}^{\prime}\left(y_{k}\right) g_{k}\left(y_{k}\right)\right|=\text { const } \quad \text { for all } y_{k}, \quad k=1, \ldots, N \text {. }
$$

Set

$$
\begin{aligned}
c_{s} & :=\left\|s \rho_{s} / \rho\right\|_{\infty} ; \\
c_{k}^{\prime} & = \begin{cases}\left\|\rho_{k} / \rho\right\|_{\infty} & \text { if } \Omega_{k}=\mathbb{R} \\
0 & \text { otherwise } \\
0 & \text { if } \Omega_{k}=\mathbb{R} \\
\left\|y_{k} \rho_{k} / \rho\right\|_{\infty} & \text { otherwise }\end{cases}
\end{aligned}
$$

We get, assuming that $\gamma_{1} \neq 0$ :

$$
\begin{aligned}
h_{\sigma}^{\prime}:= & \sum_{k=1}^{N}\left(c_{s}\left|y_{k}\right|^{\gamma_{k}} s^{\beta_{k}-1}+\nu_{k} c_{k}^{\prime}\left|y_{k}\right|^{1-\gamma_{k}}\right. \\
& \left.+\nu_{k} c_{k}^{\prime \prime}\left|y_{k}\right|^{-\gamma_{k}}+\beta_{k}\left|y_{k}\right|^{\gamma_{k}} s^{\beta_{k}-1}+\left(1-\gamma_{k}\right) \nu_{k}\left|y_{k}\right|^{-\gamma_{k}}\right),
\end{aligned}
$$

$$
\begin{aligned}
& \hat{h}_{\eta}^{\prime}:=\sum_{k=1}^{N}\left(\beta_{k}\left|y_{k}\right|^{\gamma_{k}} s^{\beta_{k}-1}+\left(1-\gamma_{k}\right) \nu_{k}\left|y_{k}\right|^{-\gamma_{k}}\right), \\
& \tilde{h}_{\eta}^{\prime}:=\sum_{k=1}^{N}\left(\frac{\theta_{k}\left|\mu_{k}-y_{k}\right|}{\nu_{k}\left|y_{k}\right|^{1-\gamma_{k}}}+\frac{r\left|y_{1}\right|^{\gamma_{1}}}{\gamma_{1} \nu_{1}}\right) .
\end{aligned}
$$

Therefore when all $y_{k} \in \mathbb{R}$, we can choose $h^{\prime}$ as

$$
\begin{aligned}
h^{\prime}:= & 1+\sum_{k=1}^{N}\left(\left|y_{k}\right|^{\gamma_{k}}\left(1+s^{\beta_{k}-1}\right)+\left(1-\gamma_{k}\right)\left|y_{k}\right|^{-\gamma_{k}}+\left|y_{k}\right|^{\gamma_{k}-1}\right) \\
& +\sum_{k \in I}^{k}\left|y_{k}\right|^{1-\gamma_{k}}+\sum_{k \in J}\left|y_{k}\right|^{-\gamma_{k}} .
\end{aligned}
$$

Without the commutator analysis we would get

$$
\begin{aligned}
\hat{h}_{\eta} & :=\sum_{k=1}^{N}\left(\beta_{k}\left|y_{k}\right|^{\gamma_{k}} s^{\beta_{k}-1}+\nu_{k}\left|\hat{\kappa}_{k}\right|\left|y_{k}\right|^{-\gamma_{k}}+\left(1-\gamma_{k}\right) \nu_{k}\left|y_{k}\right|^{-\gamma_{k}}\right), \\
\tilde{h}_{\eta} & :=\sum_{k=1}^{N}\left(\theta_{k} \frac{\left|\mu_{k}-y_{k}\right|}{\nu_{k}\left|y_{k}\right|^{1-\gamma_{k}}}+r s^{1-\beta_{1}}\left|y_{1}\right|^{-\gamma_{1}}\right) .
\end{aligned}
$$


Therefore we can choose

$$
h:=h^{\prime \prime} ; \quad h^{\prime \prime}:=h^{\prime}+r s^{1-\beta_{1}} /\left|y_{1}\right|^{\gamma_{1}}+\sum_{k} \nu_{k}\left|\hat{\kappa}_{k}\right|\left|y_{k}\right|^{-\gamma_{k}} .
$$

So, we always have that $h^{\prime} \leq h^{\prime \prime}$, meaning that it is advantageous to use the commutator analysis, due to the term $r s^{1-\beta_{1}} /\left|y_{1}\right|^{\gamma_{1}}$ above in particular. The last term in the above r.h.s. has as contribution only when $\gamma_{k} \neq 1$ (since otherwise $h^{\prime}$ includes a term of the same order).

6.2. Application to the VAT model. For the variant of the Achdou and Tchou model with multiple factors (VAT), i.e. when $\gamma_{k}=1$, for $k=1$ to $N$, we can take $h$ equal to

$$
h_{T A}^{\prime}:=1+\sum_{k=1}^{N}\left|y_{k}\right|\left(1+s^{\beta_{k}-1}\right),
$$

when the commutator analysis is used, and when it is not, take $h$ equal to

$$
h_{T A}:=h_{T A}+r s^{1-\beta_{1}}\left|y_{1}\right|^{-1}+\sum_{k=1}^{N} \nu_{k}\left|\hat{\kappa}_{k}\right|\left|y_{k}\right|^{-1} \text {. }
$$

Remember that $u_{T}(s)=(s-K)_{+}$for a call option, and $u_{T}(s)=(K-s)_{+}$for a put option, both with strike $K>0$.

LEMMA 6.1. For the VAT model, using the commutator analysis, in case of a call (resp. put) option with strike $K>0$, we can take $\rho=\rho_{\text {call }},\left(\right.$ resp. $\left.\rho=\rho_{\text {put }}\right)$, with

$$
\begin{aligned}
& \rho_{\text {call }}(s, y):=\left(1+s^{3+\varepsilon^{\prime \prime}}\right)^{-1} \Pi_{k=1}^{N} e^{-\varepsilon N\left(y_{k}\right)}, \\
& \rho_{\text {put }}(s, y):=\frac{s^{\alpha_{P}}}{1+s^{\alpha_{P}}} \Pi_{k=1}^{N} e^{-\varepsilon N\left(y_{k}\right)},
\end{aligned}
$$

where $\alpha_{P}:=\left(\varepsilon^{\prime}+2 \sum_{k=1}^{N}\left(1-\beta_{k}\right)-1\right)_{+}$.

Proof. (i) In the case of a call option, we have that

$$
1 \geq c_{0} s^{\beta_{k}-1} \text { for } c_{0}>0 \text { small enough over the domain of integration, }
$$

so that we can as well take

$$
h(s, y)=1+\sum_{k=1}^{N}\left|y_{k}\right| \leq \Pi_{k=1}^{N}\left(1+\left|y_{k}\right|\right) .
$$

So, we need that $\varphi(s, y) \in L^{1, \rho}(\Omega)$, with

$$
\varphi(s, y)=h^{2}(s, y) u_{T}^{2}(s)=(s-K)_{+}^{2} \Pi_{k=1}^{N}\left(1+\left|y_{k}\right|\right)^{2} .
$$

By lemma 4.2, where here $J=\{0\}$ and $I=\{1, \ldots, N\}$, we may take resp.

$$
\gamma_{0}^{\prime}=2, \gamma_{0}^{\prime \prime}=0, \gamma_{k}^{\prime}>0, \gamma_{k}^{\prime \prime}>0, \quad k=1, \ldots, N,
$$

and so we may choose for $\varepsilon^{\prime}>0$ and $\varepsilon^{\prime \prime}>0$ :

$$
\alpha_{0}^{\prime}=0, \alpha_{0}^{\prime \prime}=3+\varepsilon^{\prime \prime}, \alpha_{k}^{\prime}=\varepsilon^{\prime}, \alpha_{k}^{\prime \prime}=\varepsilon^{\prime \prime}, \quad k=1, \ldots, N,
$$


so that setting $\varepsilon:=\varepsilon^{\prime}+\varepsilon^{\prime \prime}$, we can take $\rho=\rho_{\text {call }}$.

(ii) For a put option with strike $K>0,1 \leq c_{0} s^{\beta_{k}-1}$ for big enough $c_{0}>0$, over the domain of integration, so that we can as well take

$$
h(s, y)=1+\sum_{k=1}^{N}\left|y_{k}\right| s^{\beta_{k}-1} \leq \Pi_{k=1}^{N}\left(1+\left|y_{k}\right| s^{\beta_{k}-1}\right)^{2} \leq \Pi_{k=1}^{N} s^{2 \beta_{k}-2}\left(1+\left|y_{k}\right|\right)^{2}
$$

and

$$
\varphi(s, y)=h^{2}(s, y) u_{T}^{2}(s) \leq(K-s)_{+}^{2} \Pi_{k=1}^{N} s^{2 \beta_{k}-2}\left(1+\left|y_{k}\right|\right)^{2} .
$$

By lemma 4.2, in the case of a put option and since $(K-s)_{+}^{2}$ is bounded, we can take $\gamma_{k}^{\prime}, \gamma_{k}^{\prime \prime}, \alpha_{k}^{\prime}, \alpha_{k}^{\prime \prime}$ as before, for $k=1$ to $N$, and

$$
\gamma_{0}^{\prime}=0, \gamma_{0}^{\prime \prime}=2 \sum_{k=1}^{N}\left(1-\beta_{k}\right), \alpha_{0}^{\prime}=\left(\varepsilon^{\prime}+2 \sum_{k=1}^{N}\left(1-\beta_{k}\right)-1\right)_{+}, \alpha_{0}^{\prime \prime}=0
$$

the result follows.

REMARK 6.2. If we do not use the commutator analysis, then we have a greater " $h$ " function; we can check that our previous choice of $\rho$ does not apply any more (so we should consider a smaller weight function, but we do not need to make it explicit). And indeed, we have then a singularity when say $y_{1}$ is close to zero so that the previous choice of $\rho$ makes the $p$ integral undefined.

6.3. Application to the GMH model. For the generalized multiple factor Heston model (GMH), i.e. when $\gamma_{k}=1 / 2, k=1$ to $N$, we can take $h$ equal to

$$
h_{H}^{\prime}:=1+\sum_{k=1}^{N}\left(\left|y_{k}\right|^{\frac{1}{2}}\left(1+s^{\beta_{k}-1}\right)+\left|y_{k}\right|^{-\frac{1}{2}}\right),
$$

when the commutator analysis is used, and when it is not, take $h$ equal to

$$
h_{H}:=h_{H}+r s^{1-\beta_{1}}\left|y_{1}\right|^{-\frac{1}{2}} .
$$

Lemma 6.3. (i) For the GMH model, using the commutator analysis, in case of $a$ call option with strike $K$, meaning that $u_{T}(s)=(s-K)_{+}$, we can take $\rho=\rho_{\text {call }}$, with

$$
\rho_{\text {call }}(s, y):=\left(1+s^{\varepsilon^{\prime \prime}+3}\right)^{-1} \Pi_{k=1}^{N} y_{k}^{\varepsilon^{\prime}}\left(1+y_{k}^{\varepsilon+2}\right)^{-1} .
$$

(ii) For a put option with strike $K>0$, we can take $\rho=\rho_{\text {put }}$, with

$$
\rho_{\text {put }}(s, y):=\Pi_{k=1}^{N} y_{k}^{\varepsilon^{\prime}}\left(1+y_{k}^{\varepsilon+2}\right)^{-1} .
$$

Proof. (i) For the call option, using (6.14) we see that we can as well take

$$
h(s, y) \leq 1+\sum_{k=1}^{N}\left(y_{k}^{1 / 2}+y_{k}^{-1 / 2}\right) \leq(s-K)_{+}^{2} \Pi_{k=1}^{N}\left(1+y_{k}^{1 / 2}+y_{k}^{-1 / 2}\right) .
$$

So, we need that $\varphi(s, y) \in L^{1, \rho}(\Omega)$, with

$$
\varphi(s, y)=h^{2}(s, y) u_{T}^{2}(s)=(s-K)_{+}^{2} \Pi_{k=1}^{N}\left(1+y_{k}^{1 / 2}+y_{k}^{-1 / 2}\right) .
$$


By lemma 4.2 , where here $J=\{0, \ldots, N\}$, we may take resp.

$$
\gamma_{0}^{\prime}=2, \gamma_{0}^{\prime \prime}=0, \gamma_{k}^{\prime}=1, \gamma_{k}^{\prime \prime}=1, \quad k=1, \ldots, N,
$$

and so we may choose for $\varepsilon^{\prime}>0$ and $\varepsilon^{\prime \prime}>0$ :

$$
\alpha_{0}^{\prime}=0, \alpha_{0}^{\prime \prime}=3+\varepsilon^{\prime \prime}, \alpha_{k}^{\prime}=\varepsilon^{\prime}, \alpha_{k}^{\prime \prime}=\varepsilon^{\prime \prime}+2, \quad k=1, \ldots, N,
$$

so that setting $\varepsilon:=\varepsilon^{\prime}+\varepsilon^{\prime \prime}$, we can take $\rho=\rho_{\text {call }}$.

(ii) For a put option with strike $K>0,1 \leq c_{0} s^{\beta_{k}-1}$ for big enough $c_{0}>0$, over the domain of integration, so that we can as well take

$$
h(s, y)=1+\sum_{k=1}^{N}\left|y_{k}\right| s^{\beta_{k}-1} \leq \Pi_{k=1}^{N}\left(1+\left|y_{k}\right| s^{\beta_{k}-1}\right)^{2} \leq \Pi_{k=1}^{N} s^{2 \beta_{k}-2}\left(1+\left|y_{k}\right|\right)^{2}
$$

and

$$
\varphi(s, y)=h^{2}(s, y) u_{T}^{2}(s) \leq(K-s)_{+}^{2} \Pi_{k=1}^{N} s^{2 \beta_{k}-2}\left(1+\left|y_{k}\right|\right)^{2} .
$$

By lemma 4.2, in the case of a put option and since $(K-s)_{+}^{2}$ is bounded, we can take $\gamma_{k}^{\prime}, \gamma_{k}^{\prime \prime}, \alpha_{k}^{\prime}, \alpha_{k}^{\prime \prime}$ as before, for $k=1$ to $N$, and

$$
\gamma_{0}^{\prime}=0, \gamma_{0}^{\prime \prime}=0, \alpha_{0}^{\prime}=0, \alpha_{0}^{\prime \prime}=0 .
$$

the result follows.

REMARK 6.4. If we do not use the commutator analysis, then, again, we have a greater " $h$ " function; we can check that our previous choice of $\rho$ does not apply any more (so we should consider a smaller weight function, but we do not need to make it explicit). And indeed, by the behaviour of the integral for large $s$ the previous choice of $\rho$ makes the $p$ integral undefined.

Appendix A. Regularity results by Lions and Magenes [15, Ch. 1].

Let $H$ be a Hilbert space identified with its dual and scalar product denoted by $(\cdot, \cdot)$. Let $V$ be a Hilbert space, densely and continuously embedded in $H$, with duality product denoted by $\langle\cdot, \cdot\rangle_{V}$. Set

$$
W(0, T):=\left\{u \in L^{2}(0, T ; V) ; \dot{u} \in L^{2}\left(0, T ; V^{*}\right)\right\} .
$$

It is known [15, Ch. 1] that

$$
W(0, T) \subset C(0, T ; H) \quad \text { with continuous inclusion, }
$$

and that for any $u, v$ in $W(0, T)$, and $0 \leq t<t^{\prime} \leq T$, the following integration by parts formula holds:

$$
\int_{t}^{t^{\prime}}\left(\langle\dot{u}(s), v(s)\rangle_{V}+\langle\dot{v}(s), u(s)\rangle_{V}\right) \mathrm{d} s=\left(u\left(t^{\prime}\right), v\left(t^{\prime}\right)\right)_{H}-(u(t), v(t))_{H} .
$$

Equivalently,

$$
2 \int_{t}^{t^{\prime}}\langle\dot{u}(s), u(s)\rangle_{V} \mathrm{~d} s=\left\|u\left(t^{\prime}\right)\right\|_{H}^{2}-\|u(t)\|_{H}^{2}, \quad \text { for all } u \in W(0, T) .
$$


Let $A(t) \in L^{\infty}\left(0, T ; L\left(V, V^{*}\right)\right)$ satisfy the hypotheses of uniform continuity and semicoercivity, i.e., for some $\alpha>0, \lambda \geq 0$, and $c>0$ :

$$
\left\{\begin{array}{l}
\langle A(t) u, u\rangle_{V} \geq \alpha\|u\|_{V}^{2}-\lambda\|u\|_{H}, \quad \text { for all } u \in V, \text { and a.a. } t \in[0, T], \\
\|A(t) u\|_{V^{*}} \leq c\|u\|_{V}, \quad \text { for all } u \in V, \text { and a.a. } t \in[0, T] .
\end{array}\right.
$$

Given $\left(f, u_{T}\right) \in L^{2}\left(0, T ; V^{*}\right) \times H$, we consider the following (backward) parabolic equation: find $u$ in $W(0, T)$ such

$$
\left\{\begin{aligned}
-\dot{u}(t)+A(t) u(t) & =f & & \text { in } L^{2}\left(0, T ; V^{*}\right), \\
u(T) & =u_{T} & & \text { in } H
\end{aligned}\right.
$$

and recall classical results from [15, Ch. 1].

Proposition A.1 (first parabolic estimate). The parabolic equation (A.6) has a unique solution $u \in W(0, T)$, and for some $c>0$ not depending on $\left(f, u_{T}\right)$ :

$$
\|u\|_{L^{2}(0, T ; V)}+\|u\|_{L^{\infty}(0, T ; H)} \leq c\left(\left\|u_{T}\right\|_{H}+\|f\|_{L^{2}\left(0, T ; V^{*}\right)}\right) .
$$

We next derive a stronger result with the hypothesis of semi-symmetry below: (A.8)

$A(t)=A_{0}(t)+A_{1}(t), A_{0}(t)$ and $A_{1}(t)$ continuous linear mappings $V \rightarrow V^{*}$, $A_{0}(t)$ symmetric and continuously differentiable $V \rightarrow V^{*}$ w.r.t. $t$,

$A_{1}(t)$ is measurable with range in $H$, and for positive numbers $\alpha_{0}, c_{A, 1}$ :

(i) $\left\langle A_{0}(t) u, u\right\rangle_{V} \geq \alpha_{0}\|u\|_{V}^{2}$, for all $u \in V$, and a.a. $t \in[0, T]$,

(ii) $\left\|A_{1}(t) u\right\|_{H} \leq c_{A, 1}\|u\|_{V}$, for all $u \in V$, and a.a. $t \in[0, T]$, $f \in L^{2}(0, T ; H)$ and $u_{T} \in V$.

Proposition A.2 (second parabolic estimate). Let (A.8) hold. Then the solution $u \in W(0, T)$ of (A.6) belongs to $L^{\infty}(0, T ; V)$, $\dot{u}$ belongs to $L^{2}(0, T ; H)$, and for some $c>0$ not depending on $\left(f, u_{T}\right)$ :

$$
\|u\|_{L^{\infty}(0, T ; V)}+\|\dot{u}\|_{L^{2}(0, T ; H)} \leq c\left(\left\|u_{T}\right\|_{V}+\|f\|_{L^{2}(0, T ; H)}\right) .
$$

Appendix B. Parabolic variational inequalities.

Let $K \subset V$ be a non-empty, closed and convex set, $\mathcal{K}$ be the closure of $K$ in $H$, and $u_{T} \in K$. Let

$$
\left\{\begin{array}{l}
L^{2}(0, T ; K):=\left\{u \in L^{2}(0, T ; V) ; u(t) \in K \text { a.e. }\right\} \\
W(0, T ; K):=W(0, T) \cap L^{2}(0, T ; K) .
\end{array}\right.
$$

We consider parabolic variational inequalities as follows: find $u \in W(0, T ; K)$ such that

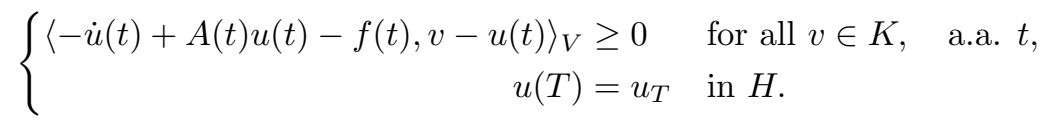

Take $v \in W(0, T ; K)$. Adding to the previous inequality the integration by parts formula

$$
-\int_{0}^{T}\langle\dot{v}(s)-\dot{u}(s), v(s)-u(s)\rangle_{V} \mathrm{~d} s=\frac{1}{2}\|u(0)-v(0)\|_{H}^{2}-\frac{1}{2}\|u(T)-v(T)\|_{H}^{2}
$$


and since $u(T)=u_{T}$ we find that

(B.4)

$$
\left\{\begin{array}{l}
\int_{0}^{T}\langle-\dot{v}(t)+A(t) u(t)-f(t), v-u(t)\rangle_{V} \geq \frac{1}{2}\|u(0)-v(0)\|_{H}^{2}-\frac{1}{2}\|u(T)-v(T)\|_{H}^{2} \\
\quad \text { for all } v \in W(0, T ; K), \quad u(T)=u_{T} .
\end{array}\right.
$$

It can be proved that the two formulation (B.2) and (B.4) are equivalent (they have the same set of solutions), and that they have at most one solution. The weak formulation is as follows: find $u \in L^{2}(0, T ; K) \cap C(0, T ; H)$ such that

$$
\left\{\begin{array}{l}
\int_{0}^{T}\langle-\dot{v}(t)+A(t) u(t)-f(t), v-u(t)\rangle_{V} \geq-\frac{1}{2}\|u(T)-v(T)\|_{H}^{2} \\
\quad \text { for all } v \in L^{2}(0, T ; K), \quad u(T)=u_{T} .
\end{array}\right.
$$

Clearly a solution of the strong formulation (B.2) is solution of the weak one.

Proposition B.1 (Brézis [6]). The following holds:

(i) Let $u_{T} \in \mathcal{K}$ and $f \in L^{2}\left(0, T ; V^{*}\right)$. Then the weak formulation (B.5) has a unique solution $u$ and, for some $c>0$, given $v_{0} \in K$ :

$$
\|u\|_{L^{\infty}(0, T ; H)}+\|u\|_{L^{2}(0, T ; V)} \leq c\left(\left\|u_{T}\right\|_{H}+\|f\|_{L^{2}\left(0, T ; V^{*}\right)}+\left\|v_{0}\right\|_{V}\right) .
$$

(ii) Let in addition the semi-symmetry hypothesis (A.8) hold, and let $u_{T}$ belong to $K$. Then $u \in L^{\infty}(0, T ; V), \dot{u} \in L^{2}(0, T ; H)$, and $u$ is the unique solution of the original formulation (B.2). Furthermore, for some $c>0$ :

$$
\|u\|_{L^{\infty}(0, T ; V)}+\|\dot{u}\|_{L^{2}(0, T ; H)} \leq c\left(\left\|u_{T}\right\|_{V}+\|f\|_{L^{2}(0, T ; H)}\right) .
$$

Appendix C. Monotonicity. Assume that $H$ is an Hilbert lattice, i.e., is endowed with an order relation $\succeq$ compatible with the vector space structure:

$$
x_{1} \succeq x_{2} \text { implies that } \gamma x_{1}+x \succeq \gamma x_{2}+x \text {, for all } \gamma \geq 0 \text { and } x \in H \text {, }
$$

such that the maxima and minima denoted by $\max \left(x_{1}, x_{2}\right)$ and $\min \left(x_{1}, x_{2}\right)$ are well defined, the operator max, min be continuous, with $\min \left(x_{1}, x_{2}\right)=-\max \left(-x_{1},-x_{2}\right)$. Setting $x_{+}:=\max (x, 0)$ and $x_{-}:=-\min (x, 0)$ we have that $x=x_{+}-x_{-}$. Assuming that the maximum of two elements of $V$ belong to $V$ we see that we have an induced lattice structure on $V$. The induced dual order over $V^{*}$ is as follows: for $v_{1}^{*}$ and $v_{2}^{*}$ in $V^{*}$, we say that $v_{1}^{*} \geq v_{2}^{*}$ if $\left\langle v_{1}^{*}-v_{2}^{*}, v\right\rangle_{V} \geq 0$ whenever $v \geq 0$.

Assume that we have the following extension of the integration by parts formula (B.3): for all $u, v$ in $W(0, T)$ and $0 \leq t<t^{\prime} \leq T$,

$$
2 \int_{t}^{t^{\prime}}\left\langle\dot{u}(s), u_{+}(s)\right\rangle_{V} \mathrm{~d} s=\left\|u_{+}\left(t^{\prime}\right)\right\|_{H}^{2}-\left\|u_{+}(t)\right\|_{H}^{2} .
$$

and that

$$
\left\langle A(t) u, u_{+}\right\rangle_{V}=\left\langle A(t) u_{+}, u_{+}\right\rangle_{V} .
$$

Proposition C.1. Let $u_{i}$ be solution of the parabolic equation (A.6) for $\left(f, u_{T}\right)=$ $\left(f^{i}, u_{T}^{i}\right), i=1,2$. If $f^{1} \geq f 2$ and $u_{T}^{1} \geq u_{T}^{2}$, then $u_{1} \geq u_{2}$. 
This type of result may be extended to the case of variational inequalities. If $K$ and $K^{\prime}$ are two subsets of $V$, we say that $K$ dominates $K^{\prime}$ if for any $u \in K$ and $u^{\prime} \in K^{\prime}, \max \left(u, u^{\prime}\right) \in K$ and $\min \left(u, u^{\prime}\right) \in K^{\prime}$.

Proposition C.2. Let $u_{i}$ be solution of the weak formulation (B.5) of the parabolic variational inequality for $\left(f, u_{T}, K\right)=\left(f^{i}, u_{T}^{i}, K^{i}\right), i=1,2$. If $f_{1} \geq f_{2}, u_{T}^{1} \geq u_{T}^{2}$, and $K^{1}$ dominates $K^{2}$, then $u_{1} \geq u_{2}$.

The monotonicity w.r.t. the convex $K$ is due to Haugazeau [13] (in an elliptic setting, but the result is easily extended to the parabolic one). See also Brézis [7].

Appendix D. Link with American options. An American option is the right to get a payoff $\Psi(t, x)$ at any time $t<T$ and $u_{T}$ at time $T$. We can motivate as follows the derivation of the associated variational inequalities. If the option can be exercized only at times $t_{k}=h k$, with $h=T / M$ and $k=0$ to $M$ (Bermudean option), then the same PDE as for the European option holds over $\left(t_{k}, t_{k+1}\right), k=0$ to $M-1$. Denoting by $\tilde{u}_{k}$ the solution of this PDE, we have that $u\left(t_{k}\right)=\max \left(\Psi, \tilde{u}_{k}\right)$. Assuming that $A$ does not depend on time and that there is a flux $f(t, x)$ of dividents, we compute the approximation $u_{k}$ of $u\left(t_{k}\right)$ as follows. Discretizing the PDE with the implicit Euler scheme we obtain the continuation value $\hat{u}_{k}$ solution of

$$
\frac{\hat{u}_{k}-u_{k+1}}{h}+A \hat{u}_{k}=f\left(t_{k}, \cdot\right), k=0, \ldots, M-1 ; \quad u_{M}=\max (\Psi, 0),
$$

so that $u_{k}=u_{k+1}-h A \hat{u}_{k}+h f\left(t_{k}, \cdot\right)$, we find that

$$
u_{k}=\max \left(\hat{u}_{k}, \Psi\right)=\max \left(u_{k+1}-h A \hat{u}_{k}+h f\left(t_{k}, \cdot\right), \Psi\right),
$$

which is equivalent to

$$
\min \left(u_{k}-\Psi, \frac{u_{k}-u_{k+1}}{h}+A \hat{u}_{k}-f\left(t_{k}, \cdot\right)\right)=0 .
$$

This suggest for the continuous time model and general operators $A$ and r.h.s. $f$ the following formulation

(D.4) $\min (u(t, x)-\Psi(x),-\dot{u}(t, x)+A(t, x) u(t, x)-f(t, x))=0, \quad(t, x) \in(0, T) \times \Omega$.

The above equation has a rigorous mathematical sense in the context of viscosity solution, see Barles [5]. However we rather need the variational formulation which can be derived as follows. Let $v(x)$ satisfy $v(x) \geq \Psi(x)$ a.e., be smooth enough. Then

$$
\begin{aligned}
& \left.\int_{\Omega}(-\dot{u}(t, x)+A(t, x) u(t, x)-f(t, x))\right)(v(x)-u(t, x)) \mathrm{d} x= \\
& \left.\int_{\{u(t, x)=\Psi(x)\}}(-\dot{u}(t, x)+A(t, x) u(t, x)-f(t, x))\right)(v(x)-u(t, x)) \mathrm{d} x \\
& \left.+\int_{\{u(t, x)>\Psi(x)\}}(-\dot{u}(t, x)+A(t, x) u(t, x)-f(t, x))\right)(v(x)-u(t, x)) \mathrm{d} x .
\end{aligned}
$$

The first integrand is nonnegative, being a product of nonnegative terms, and the second integrand is equal to 0 since by (D.3), $-\dot{u}(t, x)+A(t, x) u(t, x)-f(t, x))=0$ a.e. when $u(t, x)>\Psi(x)$. So we have that, for all $v \geq \Psi$ smooth enough:

$$
\left.\int_{\Omega}(-\dot{u}(t, x)+A(t, x) u(t, x)-f(t, x))\right)(v(x)-u(t, x)) \mathrm{d} x \geq 0 .
$$


We see that this is of the same nature as a parabolic variational inequality, where $K$ is the set of functions greater or equal to $\Psi$ (in an appropriate Sobolev space).

Appendix E. Some one dimensional problems. It is not always easy to characterize the space $\mathcal{V}$. Let us give a detailed analysis in a simple case.

E.1. The Black-Scholes setting. For the Black-Scholes model with zero interest rate (the extension to a constant nonzero interest rate is easy) and unit volatility coefficient, we have that $A u=-\frac{1}{2} x^{2} u^{\prime \prime}(x)$, with $x \in(0, \infty)$. In the case of a put option: $u_{T}(x)=(K-x)_{+}$we may take $H:=L^{2}\left(\mathbb{R}_{+}\right)$. For $v \in \mathcal{D}(0, \infty)$ and $u$ sufficiently smooth we have that $-\frac{1}{2} \int_{0}^{\infty} x^{2} u^{\prime \prime}(x) \mathrm{d} x=a(u, v)$ with

$$
a(u, v):=\frac{1}{2} \int_{0}^{\infty} x^{2} u^{\prime}(x) v^{\prime}(x) \mathrm{d} x+\int_{0}^{\infty} x u^{\prime}(x) v(x) \mathrm{d} x .
$$

This bilinear form $a$ is continuous and semi coercive over the set

$$
V:=\left\{u \in H ; x u^{\prime}(x) \in H\right\} .
$$

It is easily checked that $\bar{u}(x):=x^{-1 / 3} /(1+x)$ belongs to $V$. So, some elements of $V$ are unbounded near zero.

We now claim that $\mathcal{D}(0, \infty)$ is a dense subset of $V$. First, it follows from a standard truncation argument and the dominated convergence theorem that $V_{\infty}:=$ $V \cap L^{\infty}(0, \infty)$ is a dense subset of $V$. Note that elements of $V$ are continuous over $(0, \infty)$. Given $\varepsilon>0$ and $u \in V_{\infty}$, define

$$
u_{\varepsilon}(x):= \begin{cases}0 & \text { if } x \in(0, \varepsilon), \\ u(2 \varepsilon)(x / \varepsilon-1) & \text { if } x \in[\varepsilon, 2 \varepsilon], \\ u(2 \varepsilon) & \text { if } x>2 \varepsilon\end{cases}
$$

Obviously $u_{\varepsilon} \in V_{\infty}$. By the dominated convergence theorem, $u_{\varepsilon} \rightarrow u$ in $H$. Set for $w \in V$

$$
\Phi_{\varepsilon}(w):=\int_{0}^{2 \varepsilon} x^{2} w^{\prime}(x)^{2} \mathrm{~d} x .
$$

Since $\Phi_{\varepsilon}$ is quadratic and $v_{\varepsilon} \rightarrow u$ in $H$, we have that:

$$
\frac{1}{2} \int_{0}^{\infty} x^{2}\left(u_{\varepsilon}^{\prime}-u^{\prime}\right)^{2} \mathrm{~d} x=\frac{1}{2} \Phi_{\varepsilon}\left(u_{\varepsilon}-u\right) \leq \Phi_{\varepsilon}\left(u_{\varepsilon}\right)+\Phi_{\varepsilon}(u) .
$$

Since $u \in V, \Phi_{\varepsilon}(u) \rightarrow 0$ and

$$
\Phi_{\varepsilon}\left(u_{\varepsilon}\right) \leq\|u\|_{\infty}^{2} \int_{0}^{2 \varepsilon} \varepsilon^{-2} x^{2} \mathrm{~d} x=O\left(\|u\|_{\infty}^{2} \varepsilon\right) .
$$

So, the l.h.s. of (E.5) has limit 0 when $\varepsilon \downarrow 0$. We have proved that the set $V^{0}$ of functions in $V^{\infty}$ equal to zero near zero, is a dense subset of $V$. Now define for $N>0$

$$
\varphi_{N}(x)= \begin{cases}1 & \text { if } x \in(0, N), \\ 1-\log (x / N) & \text { if } x \in[N, e N], \\ 0 & \text { if } x>e N\end{cases}
$$

Given $u \in V_{0}$, set $u_{N}:=u \varphi_{N}$. Then $u_{N} \in H$ and, by a dominated convergence argument, $u_{N} \rightarrow u$ in $H$. The weak derivative of $u_{N}$ is $u_{N}^{\prime}=u^{\prime} \varphi_{N}+u \varphi_{N}^{\prime}$. By a 
dominated convergence argument, $x u^{\prime} \varphi_{N} \rightarrow x u^{\prime}$ in $L^{2}\left(\mathbb{R}_{+}\right)$. It remains to prove that $x u \varphi_{N}^{\prime} \rightarrow 0$ in $L^{2}\left(\mathbb{R}_{+}\right)$. But $\varphi_{N}^{\prime}$ is equal to $1 / x$ over its support, so that

$$
\left\|x u \varphi_{N}^{\prime}\right\|_{L^{2}\left(\mathbb{R}_{+}\right)}^{2}=\int_{N}^{e N} u^{2}(x) \mathrm{d} x \leq \int_{N}^{\infty} u^{2}(x) \mathrm{d} x \rightarrow 0
$$

when $N \uparrow \infty$. The claim is proved.

E.2. The CIR setting. In the Cox-Ingersoll-Ross model [8] the stochastic process satisfies

$$
\mathrm{d} s(t)=\theta(\mu-s(t)) \mathrm{d} t+\sigma \sqrt{s} \mathrm{~d} W(t), \quad t \geq 0
$$

We assume the coefficients $\theta, \mu$ and $\sigma$ to be constant and positive. The associated $\mathrm{PDE}$ is given by

$$
\left\{\begin{aligned}
A u:=-\theta(\mu-x) u^{\prime}-\frac{1}{2} x \hat{\sigma}^{2} u^{\prime \prime} & =0 & & (x, t) \in \mathbb{R}^{+} \times(0, T), \\
u(x, T) & =u_{T}(x) & & x \in \mathbb{R}^{+} .
\end{aligned}\right.
$$

Again for the sake of simplicity we will take $\rho(x)=1$, which is well-adapted in the case of a payoff with compact support in $(0, \infty)$. For $v \in \mathcal{D}(0, \infty)$ and $u$ sufficiently smooth we have that $\int_{0}^{\infty} A u(x) v(x) \mathrm{d} x=a(u, v)$ with

$a(u, v):=\theta \int_{0}^{\infty}(\mu-x) u^{\prime}(x) v(x) \mathrm{d} x+\frac{1}{2} \hat{\sigma}^{2} \int_{0}^{\infty} x u^{\prime}(x) v^{\prime}(x) \mathrm{d} x+\frac{1}{2} \hat{\sigma}^{2} \int_{0}^{\infty} u^{\prime}(x) v(x) \mathrm{d} x$.

So one should take $\mathcal{V}$ of the form

$$
\mathcal{V}:=\left\{u \in H ; \sqrt{x} u^{\prime}(x) \in L^{2}\left(\mathbb{R}_{+}\right)\right\}
$$

We next determine $H$ by requiring that the bilinear form is continuous; by the CauchySchwarz inequality (E.13)

$\left|\int_{0}^{\infty} u^{\prime}(x) v(x) \mathrm{d} x\right| \leq\left\|x^{1 / 2} u^{\prime}\right\|_{2}\left\|x^{-1 / 2} v\right\|_{2} ; \quad\left|\int_{0}^{\infty} x u^{\prime}(x) v(x) \mathrm{d} x\right| \leq\left\|x^{1 / 2} u^{\prime}\right\|_{2}\left\|x^{1 / 2} v\right\|_{2}$.

We easily deduce that the bilinear form $a$ is continuous and semi coercive over $\mathcal{V}$, when choosing

$$
H:=\left\{v \in L^{2}\left(\mathbb{R}_{+}\right) ;\left(x^{1 / 2}+x^{-1 / 2}\right) v \in L^{2}\left(\mathbb{R}_{+}\right)\right\}
$$

Note that then the integrals below are well defined and finite for any $v \in \mathcal{V}$ :

$$
\int_{0}^{\infty}\left(x^{1 / 2} v^{\prime}\right)\left(x^{-1 / 2} v\right)=\int_{0}^{\infty} v v^{\prime}=\frac{1}{2} \int_{0}^{\infty}\left(v^{2}\right)^{\prime} .
$$

So $w:=v^{2}$ is the primitive of an integrable function and therefore has a limit at zero. Since $v$ is continuous over $(0, \infty)$ it follows that $v$ has a limit at zero.

However if this limit is nonzero we get a contradiction with the condition that $x^{-1 / 2} v \in L^{2}\left(\mathbb{R}_{+}\right)$. So, every element of $\mathcal{V}$ has zero value at zero.

We now claim that $\mathcal{D}(0, \infty)$ is a dense subset of $\mathcal{V}$. First, $\mathcal{V}_{\infty}:=\mathcal{V} \cap L^{\infty}(0, \infty)$ is a dense subset of $\mathcal{V}$. Note that elements of $\mathcal{V}$ are continuous over $(0, \infty)$. Given $\varepsilon>0$ 
and $u \in \mathcal{V}_{\infty}$, define $u_{\varepsilon}(x)$ as in (E.3). Then $u_{\varepsilon} \in \mathcal{V}_{\infty}$. By the dominated convergence theorem, $u_{\varepsilon} \rightarrow u$ in $H$. Set for $w \in \mathcal{V}$

$$
\Phi_{\varepsilon}(w):=\int_{0}^{2 \varepsilon} x w^{\prime}(x)^{2} \mathrm{~d} x .
$$

Since $\Phi_{\varepsilon}$ is quadratic and $u_{\varepsilon} \rightarrow u$ in $H$, we have that:

$$
\frac{1}{2} \int_{0}^{\infty} x^{2}\left(u_{\varepsilon}^{\prime}-u^{\prime}\right)^{2} \mathrm{~d} x=\frac{1}{2} \Phi_{\varepsilon}\left(u_{\varepsilon}-u\right) \leq \Phi_{\varepsilon}\left(u_{\varepsilon}\right)+\Phi_{\varepsilon}(u) .
$$

Since $u \in \mathcal{V}, \Phi_{\varepsilon}(u) \rightarrow 0$ and

$$
\Phi_{\varepsilon}\left(u_{\varepsilon}\right) \leq \varepsilon^{-2} u(2 \varepsilon)^{2} \int_{0}^{2 \varepsilon} x \mathrm{~d} x=2 u(2 \varepsilon)^{2} \rightarrow 0 .
$$

So, the 1.h.s. of (E.17) has limit 0 when $\varepsilon \downarrow 0$. We have proved that the set $\mathcal{V}^{0}$ of functions in $\mathcal{V}^{\infty}$ equal to zero near zero, is a dense subset of $\mathcal{V}$. Define $\varphi_{N}$ as in (E.7)

Given $u \in \mathcal{V}_{0}$, set $u_{N}:=u \varphi_{N}$. As before, $u_{N} \rightarrow u$ in $H$, is $u_{N}^{\prime}=u^{\prime} \varphi_{N}+u \varphi_{N}^{\prime}$, $x u^{\prime} \varphi_{N} \rightarrow x u$ in $L^{2}\left(\mathbb{R}_{+}\right)$, and it remains to prove that $x u \varphi_{N}^{\prime} \rightarrow 0$ in $L^{2}\left(\mathbb{R}_{+}\right)$. But $\varphi_{N}^{\prime}$ is equal to $1 / x$ over its support, so that when $N \uparrow \infty$ :

$$
\left\|x^{1 / 2} u \varphi_{N}^{\prime}\right\|_{L^{2}\left(\mathbb{R}_{+}\right)}^{2}=\int_{N}^{e N} x^{-1} u^{2}(x) \mathrm{d} x \leq \int_{N}^{\infty} u^{2}(x) \mathrm{d} x \rightarrow 0 .
$$

The claim is proved. (1)

西

[1] Y. Achdou, B. Franchi, and N. Tchou, A partial differential equation connected to option
pricing with stochastic volatility: regularity results and discretization, Math. Comp. $\mathbf{7 4}$ (2005), no. 251, 1291-1322.

[2] Y. Achdou and O. Pironneau, Computational methods for option pricing, Society for Industrial and Applied Mathematics (SIAM), Philadelphia, PA, 2005.

[3] Y. Achdou and N. Tchou, Variational analysis for the Black and Scholes equation with stochastic volatility, M2AN Math. Model. Numer. Anal. 36 (2002), no. 3, 373-395.

[4] T. Aubin, A course in differential geometry, Graduate Studies in Mathematics, vol. 27, American Mathematical Society, Providence, RI, 2001.

[5] G. Barles, Convergence of numerical schemes for degenerate parabolic equations arising in finance theory, Numerical methods in finance, Publ. Newton Inst., vol. 13, Cambridge Univ. Press, Cambridge, 1997, pp. 1-21.

[6] H. Brézis, Inéquations variationnelles paraboliques, Séminaire Jean Leray.

[7] _ Problèmes unilatéraux, Journal de Mathématiques pures et appliquées 51 (1972), 1168.

[8] J.C. Cox, J. E. Ingersoll, Jr., and S. A. Ross, A theory of the term structure of interest rates, Econometrica 53 (1985), no. 2, 385-407. MR 785475

[9] P.M. N. Feehan and C. A. Pop, Schauder a priori estimates and regularity of solutions to boundary-degenerate elliptic linear second-order partial differential equations, J. Differential Equations 256 (2014), no. 3, 895-956. MR 3128929

[10] P.M.N. Feehan and C. A. Pop, Degenerate-elliptic operators in mathematical finance and higher-order regularity for solutions to variational equations., Adv. Differ. Equ. 20 (2015), no. 3-4, 361-432 (English).

[11] J.-P. Fouque, G. Papanicolaou, and K.R. Sircar, Derivatives in financial markets with stochastic volatility, Cambridge University Press, Cambridge, 2000.

[12] P. Gauthier and D. Possamaï, Efficient simulation of the double Heston model, Tech. report, SSRN working paper series, 2009, Revised in Jan. 2010.

[13] Y. Haugazeau, Sur des inéquations variationnelles, C. R. Acad. Sci. Paris Sér. A-B 265 (1967), A95-A98. 
[14] S.L. Heston, A closed-form solution for options with stochastic volatility with applications to bond and currency options, The Review of Financial Studies 6 (1993), no. 2, 327-343.

[15] J.-L. Lions and E. Magenes, Non-homogeneous boundary value problems and applications. Vol. I, Springer-Verlag, New York, 1972, Translated from the French by P. Kenneth, Die Grundlehren der mathematischen Wissenschaften, Band 181.

[16] P.-L. Lions and M. Musiela, Correlations and bounds for stochastic volatility models, Ann. Inst. H. Poincaré Anal. Non Linéaire 24 (2007), no. 1, 1-16.

[17] K. Jacobs P. Christoffersen, S. Heston, The shape and term structure of the index option smirk: Why multifactor stochastic volatility models work so well, Management Science 55 (2009), no. 12, 1914-1932.

[18] O. Pironneau and Y. Achdou, Partial differential equations for option pricing, Handbook of numerical analysis Vol XV. Special Volume: Mathematical modeling and numerical methods in finance, Amsterdam: Elsevier/North-Holland, 2009, pp. 369-495. 